\title{
Eigenfunction Expansions Associated with the Schrödinger Operator with a Complex Potential and the Scattering Theory*
}

\author{
By \\ Kiyoshi MochizUKI ${ }^{\dagger}$
}

\section{Introduction}

The present paper is devoted to a detailed description of the results summarized in the author's preceding note [1]. The purpose of [1] was a generalization to the non-selfadjoint case of the eigenfunction expansion and the scattering theory developed by Povzner $[2,3]$, Faddeev [4], and Ikebe $[5,6]$ for the selfadjoint Schrödinger operator in the 3-dimensional Euclidean space $E_{3}$.

We shall study the operator $L$ obtained by closure in $L^{2}\left(E_{3}\right)$ of the differential operator defined by

$$
-\Delta f+q(x) f \text { for } f \in C_{0}^{\infty}\left(E_{3}\right),
$$

where $x=\left(x_{1}, x_{2}, x_{3}\right) \in E_{3}$, and $\Delta$ denotes the Laplace operator. Everywhere, unless specifically stated otherwise, the potential $q(x)$ is assumed to be a complex valued function satisfying the condition

$$
(1+|x|)^{(1+\delta) / 2} q(x) \in L^{2}\left(E_{3}\right) \quad \delta>0 .
$$

Some results concerning the spectrum of the operator $L$ can be obtained as a consequence of more general theorems concerning the index theory for closed operators investigated by Gohberg-Krein [7], Schechter [8], and others. In particular, we can show that the essential spectrum of $L$ fills the half-axis $[0, \infty)$ (Theorem 1.1).

Received July 12, 1968.

Communicated by S. Matsuura.

* This research was partly supported by the Sakkokai Foundation.

$\dagger$ Faculty of Liberal Arts, Kyoto University. 
The distorted plane waves $\varphi_{ \pm}(x, k)=\exp \{i k \cdot x\}+v_{ \pm}(x, k)$ and the adjoint waves $\phi_{ \pm}^{*}(x, k)=\exp \{i k \cdot x\}+v_{ \pm}^{*}(x, k)$ for the operator $L$ are bounded, continuous functions of $x \in E_{3}$ for each fixed $k \in E_{3}(k \neq 0)$ satisfying the Schrödinger equations

$$
\begin{aligned}
& -\Delta \varphi+q(x) \varphi=\mu \varphi \\
& -\Delta \varphi^{*}+q(x) \varphi^{*}=\mu \varphi *
\end{aligned}
$$

with $\mu=|k|^{2}$. The scattered waves $v_{ \pm}(x, k)$ and $v_{ \pm}^{*}(x, k)$ both behave like $O\left(|x|^{-1}\right)$ at infinity, where the subscripts "+" and "-" denote the incoming and outgoing waves, respectively.

So far as a real potential is concerned, $\varphi_{ \pm}(x, k)=\phi_{ \pm}^{*}(x, k)$ can be found for each $\mu=|k|^{2}>0$ as unique solutions of the LippmannSchwinger equation

$$
\mathcal{P}_{ \pm}(x, k)=\exp \{i k \cdot x\}-\int_{E_{3}} \frac{\exp \left\{\mp i\left|k_{1} \cdot\right| x-y_{i}^{\prime}\right\}}{4 \pi|x-y|} q(y) \varphi_{ \pm}(y, k) d y
$$

under a condition ${ }^{1)}$ similar to (A) on $q(x)$ (see Ikebe [5], Eidus [9]). This mainly follows from the fact that the solution of (0.2) for $\mu>0$ is unique $(\varphi \equiv 0)$ if it is assumed to satisfy the (incoming or outgoing) Sommerfeld radiation condition at infinity :

$$
\phi_{I}=0\left(|x|^{-1}\right) ; \lim _{\rho \rightarrow \infty} \int_{|x|=\rho}\left|\frac{\partial \varphi_{ \pm}}{\partial|x|} \pm i \sqrt{\mu} \varphi_{ \pm}\right|^{2} d S=0 .
$$

As for a complex potential, however, this uniqueness theorem can not always be concluded except for the case of the "small perturbation" (cf. J. Schwartz [10], Pavlov [11]). Let $\sigma_{s}(L)\left[\sigma_{s}^{*}(L)\right]$ denote the set of values $\mu=0$ and $\mu>0$ for which equation (0.2) $\left[(0.2)^{*}\right]$ has non-trivial solutions satisfying the (incoming or outgoing) Sommerfeld radiation condition at infinity. We shall show that $\sigma_{s}(L)=\sigma_{s}^{*}(L)$, and is compact in $[0, \infty$ ) (Theorem 2.1), and then that the distorted plane waves $\varphi_{ \pm}(x, k)$ and the adjoint waves $\varphi_{ \pm}^{*}(x, k)$ are uniquely determined for each $|k|^{2} \notin \sigma_{s}(L)$ (Theorem 4.1).

We denote by $\Gamma$ the set of (possibly infinte) subintervals $e=(\alpha, \beta)$

1) In [5], $q(x)$ is assumed to be in $L_{\text {loc }}^{2}\left(E_{3}\right)$ and to behave like $0(|x|-2-\delta)(\delta>0)$ at infinity. Our assumption (A) is essentially the same. 
of $[0, \infty)$ whose closure does not contain any point of $\sigma_{s}(L)$. In $\S 5$, we shall derive that the weak limit

$$
E(e)=\mathrm{w}-\lim _{\varepsilon \rightarrow+0} \frac{1}{2 \pi i} \int_{e}\{R(\lambda+i \varepsilon)-R(\lambda-i \varepsilon)\} d \lambda
$$

with $R(\zeta)=(L-\zeta I)^{-1}$ exists for any $e \in \Gamma$. Although $E(e)$ are not uniformly bounded in the class $\Gamma,(0.5)$ will define the so-called "spectral projection" permutable with $L$ for each fixed $e \in \Gamma$. Now the expansion formula of an arbitrary function $f(x) \in L^{2}\left(E_{3}\right)$ can be obtained in the following sense : Let $Z_{ \pm}(e)$ and $W_{ \pm}(e)$ be defined by

$$
\begin{array}{ll}
{\left[Z_{ \pm}(e) g\right](k)=(2 \pi)^{-3 / 2} \int_{E_{3}} \overline{\varphi_{ \pm}^{*}(x, k)} g(x) d x} & \left(g \in C_{0}^{\infty}\left(E_{3}\right)\right) ; \\
{\left[W_{ \pm}(e) g\right](x)=(2 \pi)^{-3 / 2} \int_{K_{e}}{\varphi_{ \pm}}(x, k) g(k) d k} & \left(g \in C_{0}^{\infty}\left(K_{e}\right)\right),
\end{array}
$$

where $K_{e}=\left\{k \in E_{3} ;|k|^{2} \in e\right\}$. Then $Z_{ \pm}(e)$ can be extended to a continuous mapping of $L^{2}\left(E_{3}\right)$ onto $L^{2}\left(K_{e}\right)$, while $W_{ \pm}(e)$ can be extended to a continuous mapping of $L^{2}\left(K_{e}\right)$ onto $E(e) L^{2}\left(E_{3}\right)$, and we have

$$
\begin{aligned}
& E(e)=W_{ \pm}(e) Z_{ \pm}(e) ; \\
& E_{0}(e)=Z_{ \pm}(e) W_{ \pm}(e),
\end{aligned}
$$

where $E_{0}(e)=E_{0, \beta}-E_{0, \infty}$ with $E_{0, \lambda}$ denoting the resolution of the identity of the selfadjoint operator $L_{0}$ determined by the expression $-\Delta$ (Theorem 5.1).

These relations with $e=(0, \infty)$ are already proved by Ikebe [5] (cf. also Povzner [3]) when $q(x)$ is a real potential. In [5], (0.9) was established with the aid of the time-dependent scattering theory. In our case, however, we can not prove this along Ikebe's line because the operators $Z_{ \pm}(e)$ and $W_{ \pm}(e)$ in $L^{2}\left(E_{3}\right)$ are not in general related to the timedependent theory. So the proof will be done by use of a different method (cf. the author [12]).

In $\S 6$, we shall discuss the time-dependent scattering theory restricting $L$ and $L_{0}$ to the invariant subspaces $E(e) L^{2}\left(E_{3}\right)$ and $E_{0}(e) L^{2}\left(E_{3}\right)$, respectively. $E(e) L^{2}\left(E_{3}\right)$ forms a Banach space with respect to the $L^{2}$-norm, and its dual space is given by $E(e)^{*} L^{2}\left(E_{3}\right)$, where 
$E(e)^{*}$ is the adjoint operator of $E(e)$. It can be shown that the operator $-i L$ acting in $E(e) L^{2}\left(E_{3}\right)$ is the infinitesimal generator of a group $\exp \{-i t L\}=W_{ \pm}(e) \exp \left\{-i t L_{0}\right\} Z_{ \pm}(e)(-\infty<t<+\infty)$ of type zero, and that $W_{ \pm}(e)$ coinside with the wave operators in the timedependent formulation (Theorem 6.1). Then the scattering operators $S(e)$ is given by (Theorem 6.2)

$$
S(e)=Z_{+}(e) W_{-}(e) .
$$

The discussion presented in $\S 6$ will be closely related to Kato [13].

Finally, in $\S 7$, we shall restrict ourselves to the case where $q(x)$ satisfies the condition

$$
(1+|x|)^{(3+\delta) / 2} q(x) \in L^{2}\left(E_{3}\right), \quad \delta>0 .
$$

Under this assumption on $q(x)$, the distorted plane waves $\varphi_{ \pm}(x, k)$ have the following asymptotic expansions for large $|x|$ :

$$
\varphi_{\perp}(x, k)=\exp \{i k \cdot x\}+\frac{\exp \{\mp i|k| \cdot|x|\}}{|x|} \theta_{ \pm}(n, \nu ;|k|)+o\left(|x|^{-1}\right),
$$

where $n=x /|x|, \nu=k /|k|$, and $\theta_{ \pm}(n, \nu ;|k|)$ is the so-called scattering amplitude. We can show that the scattering operator $S(e)$ is represented in the Fourier space as (Theorem 7.1)

$(0.12) \quad[S(e) f]^{\wedge}(|k| \nu)$

$$
=\hat{f}(|k| \nu)+\frac{i}{2 \pi} \int_{|n|=1}|k| \theta_{-}(-n,-\nu ;|k|) \hat{f}(|k| n) d n, \quad|k|^{2} \in e,
$$

and then that the potential $q(x)$ is uniquely determined by the asymptotic behavior for large $|k|$ of a given $\theta_{-}(n, \nu ;|k|)$ (Theorem 7.2). This establishes the uniqueness of the solution for the scattering inverse problem. For a given potential, we can obtain, by (0.12), the scattering matrix $S_{|k|}$ attached to a fixed value $|k|^{2}$ of the kinetic energy that is not in the compact set $\sigma_{s}(L)$. In this sense, the results obtained in this paper are of a local character. However, it may be of interest that we can determine the potential $q(x)$ from the scattering amplitude $\theta_{-}(n, \nu ;|k|)$ given only for large $|k|$. 
A different method of obtaining the scattering operator in the above form has been developed by Ikebe [6] for the real potential case. The chief concern of [6], however, is not in the investigation of the inverse problem but in the determination of the phase shift. On the other hand, the inverse problem has been studied by Faddeev [4], though relation $(0.12)$ is not proved there, when $q(x)$ (real) is additionally assumed to be a smooth function.

In conclusion, the author wishes to thank Professor T. Ikebe for his kind advices and discussions.

\section{Properties of the Resolvent}

\section{§1. Essential spectrum and the resolvent kernel.}

We shall consider the Schrödinger operator $-\Delta+q(x)$ with the complex valued potential function $q(x)$ defined on $E_{3}$, where $x$ denotes a point in $E_{3}$ with its length $|x|$. In this $\$$ we are enough to assume that $q(x)$ is square integrable $\left(q(x) \in L^{2}\left(E_{3}\right)\right)$.

Let $L_{0}$ be the selfadjoint operator in the Hilbert space $\mathfrak{N}=L^{2}\left(E_{3}\right)^{2)}$ defined by the differential expression $-\Delta$ with the domain $\mathscr{D}\left(L_{0}\right)=$ $\mathscr{D}_{L^{2}}^{2}\left(E_{3}\right)^{3)} \quad$ It is well known that $L_{0}$ has purely the continuous spectrum $[0, \infty)$. The resolvent $R_{0}(\zeta)=\left(L_{0}-\zeta I\right)^{-1}, \zeta \notin[0, \infty)$ is an integral operator with the kernel $\exp \{i \sqrt{\zeta}|x-y|\}|4 \pi| x-y \mid$, where by $\sqrt{ } \bar{\zeta}$ is meant the branch of the square root of $\zeta$ with $\operatorname{Im} \sqrt{\zeta}>0 .{ }^{4}$ Let $V$ denote the operator of multiplication by $q(x)$. As is known ([14]), $\mathscr{D}(V) \supset \mathscr{D}\left(L_{0}\right)$ under the assumption $q(x) \in L^{2}\left(E_{3}\right)$. The adjoint operator $V^{*}$ is also the multiplicative operator and is given by the complex conjugate $\overline{q(x)}$ of $q(x)$.

Lemma 1.1. Let $q(x)$ be in $L^{2}\left(E_{3}\right)$. Then the integral operator

2) We denote the inner product and the norm in $\mathfrak{S}$ by

$$
(f, g)=\int_{F_{3}} f(x) g(x) d x \text { and }\|f\|=(f, f)^{1 / 2} \text {. }
$$

3) $\mathscr{D}_{L^{2}}^{2}\left(E_{3}\right)$ is the completion of $C_{0}^{\infty}\left(E_{3}\right)$ with respect to the norm $\|f\|_{1}\|\Delta f\|$, where $C_{0}^{\infty}\left(E_{3}\right)$ consists of all functions which are infinitely differentiable and have compact supports.

4) For a complex number $\kappa, \operatorname{Im} \kappa$ and $\operatorname{Re} \kappa$ respectively mean the imaginary and real parts of $\kappa$. 
$V R_{0}(\zeta), \zeta \notin[0, \infty)$ is of Hilbert-Schmidt type. Moreover, its operator norm satisfies the inequality

$$
\left\|V R_{0}(\zeta)\right\| \leq \text { const }(\operatorname{Im} \sqrt{\zeta})^{-1 / 2}
$$

Proof. We have

$$
\begin{aligned}
& \int_{E_{3}} \int_{E_{3}}\left|\frac{q(x)\{i \sqrt{\zeta}|x-y|\}}{4 \pi|x-y|}\right|^{2} d x d y \\
\leq & (4 \pi)^{-1} \int_{E_{3}}|q(x)|^{2} d x \int_{0}^{\infty} \exp \{-2 \operatorname{Im} \sqrt{\zeta}|y|\} d|y| \\
= & (8 \pi)^{-1}|| q \|^{2}(\operatorname{Im} \sqrt{\zeta})^{-1}<+\infty .
\end{aligned}
$$

Since the norm of an integral operator does not exceed its HilbertSchmidt norm, we conclude the assertions of the lemma. Q.E.D.

Now we define the Schrödinger operator $L$ by

$$
L=L_{0}+V, \quad \mathscr{D}(L)=\mathscr{D}_{L^{2}}^{2}\left(E_{3}\right) .
$$

Then we see that $L$ is a closed operator in $\mathfrak{S}$ since the relation

$$
L-\zeta I=L_{0}-\zeta I+V=\left\{I+V R_{0}(\zeta)\right\}\left(L_{0}-\zeta I\right)
$$

is valid for any $\zeta \notin[0, \infty)$. Moreover, we have the

Lemma 1.2. (1) $L$ is the closure of the differential operator $-\Delta+q(x)$ defined initially over functions in $C_{0}^{\infty}\left(E_{3}\right)$.

(2) The adjoint operator $L^{*}$ of $L$ is given by

$$
L^{*}=L_{0}+V^{*}, \quad \mathscr{D}\left(L^{*}\right)=\mathscr{D}_{L^{2}}^{2}\left(E_{3}\right) .
$$

Proof. (1) Let $f(x) \in \mathscr{D}(L)$. Then, since $f(x) \in \mathscr{D}_{L^{2}}^{2}\left(E_{3}\right)=\mathscr{D}\left(L_{0}\right)$, there exists for any fixed $\varepsilon>0$ an $f_{\nu}(x) \in C_{0}^{\infty}\left(E_{3}\right)$ such that

$$
|| f_{\nu}-f \|+|| L_{0}\left(f_{\nu}-f\right)^{\prime} \mid<\varepsilon \text {. }
$$

On the other hand, we have for any $\zeta \notin[0, \infty)$

$$
\begin{aligned}
\left\|V\left(f_{\nu}-f\right)\right\| & \leq\left\|V R_{0}(\zeta)\right\| \cdot\left\|\left(L_{0}-\zeta I\right)\left(f_{\nu}-f\right)\right\| \\
& \leq \text { const }(\operatorname{Im} \sqrt{\zeta})^{-1 / 2}\left\{|\zeta| \cdot\left\|f_{\nu}-f\right\|+\left\|L_{0}\left(f_{\nu}-f\right)\right\|\right\}
\end{aligned}
$$

by (1.1). These inequalities imply that $\mathscr{D}_{L^{2}}^{2}\left(E_{3}\right)$ is the closure of $C_{0}^{\infty}\left(E_{3}\right)$ in the sense of the graph norm of $-\Delta+q(x)$.

(2) By virtue of (1.1), there exists a complex value $\zeta \notin[0, \infty)$ 
such that $\left\|V R_{0}(\zeta)\right\|<1$. For an arbitrary fixed such value $\zeta,(L-\zeta I)^{-1}$ exists and admits the Neumann series expansion

$$
(L-\zeta I)^{-1}=R_{0}(\zeta) \sum_{n=0}^{\infty}\left[-V R_{0}(\zeta)\right]^{n}
$$

On the other hand, since

$$
L_{0}+V^{*}-\bar{\zeta} I=\left(L_{0}-\bar{\zeta} I\right)\left\{I+R_{0}(\bar{\zeta}) V^{*}\right\} \subset\left(L_{0}-\bar{\zeta} L\right)\left\{I+\left[V R_{0}(\zeta)\right]^{*}\right\},
$$

we have similarly

$$
\left(L_{0}+V^{*}-\zeta I\right)^{-1}=\sum_{n=0}^{\infty}\left[-V R_{0}(\zeta)\right]^{* n} R_{0}(\zeta)^{*}
$$

Comparing this with (1.5), we find the relation $(L-\zeta I)^{-1 *}=$ $\left(L_{0}+V^{*}-\bar{\zeta} I\right)^{-1}$, which implies (1.4).

Q. E. D.

Following the definition given by Schechter [8], we define the essential spectrum of a closed operator as the complement in the complex plane of its Fredholm set. Here the Fredholm set of a closed operator $T$ in a Hilbert space $\mathfrak{S}$ is composed of the values $\zeta$ for which $T-\zeta I$ has the finite dimensional null space and closed range with the finite dimensional ortho-complement in $\mathfrak{S}$. Then the following theorem is a direct consequence of Theorem 3.1 of Gohberg-Krein [7] and Lemma 1.1 given above.

Theorem 1.1. The essential spectrum of $L$ fills the half-axis $[0, \infty)$, while the spectrum in the complement of $[0, \infty)$ consists of discrete eigenvalues of finite multiplicity. A value $\zeta \notin[0, \infty)$ is an eigenvalue of $L$ if and only if $\bar{\zeta}$ is an eigenvalue of $L^{*}$.

Remark 1.1. In the case where $q(x) \in L^{2}\left(E_{3}\right)$ is assumed to be continuous for large $|x|$ and behave like $o\left(|x|^{-1}\right)$ at infinity, we can say more: The residual spectrum of $L$ is empty, i.e. $(0, \infty)$ consists of the continuous spectrum, and $\zeta=0$ is either an eigenvalue or in the continuous spectrum.

Proof. By a result of Kato [15], we see that both $L$ and $L^{*}$ have no positive eigenvalues under the above conditions on $q(x)$. Since a value in the residual spectrum of $L$ is an eigenvalue of $L^{*}$, all positive numbers must be in the continuous spectrum of 
$L$. Moreover, since $\zeta=0$ becomes an eigenvalue of $L$ if and only if it is an eigenvalue of $L^{*}$, the rest of the assertion of the remark can be concluded by the same reasoning.

Q.E. D.

The above theorem shows that the resolvent $R(\zeta)=(L-\zeta I)^{-1}$ can be defined for all $\zeta \notin[0, \infty)$ outside the discrete eigenvalues of $L$. From (1.2) we have the following resolvent equation:

$$
R(\zeta)=R_{0}(\zeta)-R_{0}(\zeta) V R(\zeta)=R_{0}(\zeta)-R(\zeta) V R_{0}(\zeta) .
$$

As we proved in Lemma 1.1, $V R_{0}(\zeta)$ is an integral operator of Hilbert-Schmidt type. $R(\zeta)$ being a bounded operator, $R(\zeta) V R_{0}(\zeta)$ is also of Hilbert-Schmidt type. Since $R_{0}(\zeta)$ is known to be an integral operator of Carleman type, it follows from the third member of (1.6) that $R(\zeta)$ is of the same type, too. Let us denote by $R(x, y ; \sqrt{\zeta})$ the kernel of $R(\zeta)$. Then we have from (1.6)

$$
\begin{aligned}
R(x, y ; \sqrt{\zeta})= & \frac{\exp \{i \sqrt{\zeta}|x-y|\}}{4 \pi|x-y|} \\
& -\int_{E_{3}} R(x, z ; \sqrt{\zeta}) q(z) \frac{\exp \{i \sqrt{\zeta}|z-y|\}}{4 \pi|z-y|} d z
\end{aligned}
$$

for a.e. ${ }^{5)} y$ with a.e. fixed $x$.

We put $R^{*}(\zeta)=\left(L^{*}-\zeta I\right)^{-1}$. Then we have, corresponding to (1.6) and (1.7),

(1. 8) $R^{*}(\zeta)=R_{0}(\zeta)-R_{0}(\zeta) V^{*} R^{*}(\zeta)=R_{0}(\zeta)-R^{*}(\zeta) V^{*} R_{0}(\zeta)$,

(1.9) $R^{*}(x, y ; \sqrt{\zeta})=\frac{\exp \{i \sqrt{\zeta}|x-y|\}}{4 \pi|x-y|}$

$$
-\int_{E_{3}} R^{*}(x, z ; \sqrt{\zeta}) \overline{q(z)} \frac{\exp \{i \sqrt{\zeta}|z-y|\}}{4 \pi|z-y|} d z,
$$

where $R^{*}(x, y ; \sqrt{\zeta})$ is the kernel of $R^{*}(\zeta)$. Since

$$
R^{*}(\bar{\zeta})=R(\zeta)^{*},
$$

it follows that

(1.11) $R^{*}(x, y ; \sqrt{\bar{\zeta}})=\overline{R(y, x ; \sqrt{\zeta})}$ for a.e. $(x, y)$ in $E_{3} \times E_{3}$.

5) "a.e." means "almost every" or "almost everywhere". 
With this identity, we can follow the same line of proof as Ikebe ([5]; Theorem 1) to show the following theorem:

Theorem 1.2. (1) $R(\zeta)$ is an integral operator of Carleman type, and its kernel $R(x, y ; \sqrt{\zeta})$ satisfies the integral equation

$$
\begin{aligned}
R(x, y ; \sqrt{\zeta})= & \frac{\exp \{i \sqrt{\zeta}|x-y|\}}{4 \pi|x-y|} \\
& -\int_{E_{3}} \frac{\exp \{i \sqrt{ } \bar{\zeta}|x-z|\}}{4 \pi|x-z|} q(z) R(z, y ; \sqrt{\zeta}) d z
\end{aligned}
$$

for a.e. $(x, y)$ in $E_{3} \times E_{3}$. Moreover, $R(x, y ; \sqrt{\zeta})$ is symmetric in $x$ and $y$ :

$$
R(x, y ; \sqrt{\zeta})=R(y, x ; \sqrt{\zeta}), \quad \text { a.e. }
$$

(2) $R(x, y ; \sqrt{\zeta})$ is absolutely integrable in $y$ for a.e. fixed $x \in E_{3}$.

(2) $R^{*}(\zeta)$ has the same properties: $R^{*}(x, y ; \sqrt{\zeta})$ satisfies $(1.12)$ with $q(x)$ replaced by $\overline{q(x)}$, is symmetric in $x$ and $y$, and is absolutely integrable in $y$.

\section{§. Factorization of the potential.}

In the following $q(x)$ is assumed to be a complex valued function satisfying condition $(\mathrm{A})$ :

$$
(1+|x|)^{(1+\delta) / 2} q(x) \in L^{2}\left(E_{3}\right), \quad \delta>0 .
$$

Under this condition, $q(x) \in L^{3 / 2}\left(E_{3}\right) \cap L^{2}\left(E_{3}\right)$ since we have

$$
\begin{aligned}
\int_{E_{3}}|q(x)|^{3 / 2} d x & \leq\left[\int_{E_{3}}|q(x)|^{2}(1+|x|)^{1+\delta} d x\right]^{3 / 4} \\
& \times\left[\int_{E_{3}}(1+|x|)^{-3(1+\delta)} d x\right]^{1 / 4}<+\infty .
\end{aligned}
$$

We put $q(x)=b(x) a(x)$, where $a(x)$ is chosen as one of the following two functions :

$$
a_{1}(x)=|q(x)|^{1 / 2}, \quad \text { or } \quad a_{2}(x)=(1+|x|)^{(1+\delta) / 2} q(x) .
$$

Let $A, B$ respectively denote the operators of multiplication by $a(x), b(x)$. It should be noted that if $a(x)=a_{2}(x)$, then $a(x) \in L^{2}\left(E_{3}\right)$ 
and $b(x)=b_{2}(x)=(1+|x|)^{-(1+\delta) / 2}$ is a bounded function. Hence, in this case, $\mathscr{D}(A) \supset \mathscr{D}\left(L_{0}\right)$ and $\mathscr{D}(B)=\mathfrak{E}$. On the other hand, if $a(x)=a_{1}(x)$, then $b(x)=b_{1}(x)=[q(x) /|q(x)|] a(x)$, and in this case $\mathscr{D}(A)=\mathscr{D}(B)$ $\supset \mathscr{D}\left(L_{0}\right)$. For we have for each $f(x) \in \mathscr{D}\left(L_{0}\right)$

$$
\int_{E_{3}}|q(x)| \cdot|f(x)|^{2} d x \leq\left[\int_{E_{3}}|q(x)|^{3 / 2} d x\right]^{2 / 3}\left[\max _{x}|f(x)| \cdot|| f \|^{2}\right]^{1 / 3}<+\infty
$$

taking into account that $\mathscr{D}\left(L_{0}\right)=\mathscr{D}_{L^{2}}^{2}\left(E_{3}\right) \subset L^{\infty}\left(E_{3}\right)$. Thus, for any choice of $a(x)$, we conclude

$$
\mathscr{D}(A) \cap \mathscr{D}(B) \supset \mathscr{D}\left(L_{0}\right) .
$$

Moreover, we see easily

$$
\mathscr{D}(A) \supset B \mathscr{D}\left(L_{0}\right) \text {, and } \mathscr{D}(B) \supset A \mathscr{D}\left(L_{0}\right)
$$

for each choice of $a(x)$. Hence $V$ is a product $V=B A=A B^{6)}$ on $\mathscr{D}\left(L_{0}\right)$.

Now applying $A$ to (1.6) from the left, we have

$$
A R(\zeta)=A R_{0}(\zeta)-A R_{0}(\zeta) B A R(\zeta)=A R_{0}(\zeta)-A R(\zeta) B A R_{0}(\zeta) .
$$

Lemma 2.1. For a value $\zeta$ in the resolvent set of $L$, if there exists a bounded operator $T(\zeta)$ satisfying the equation

$$
T(\zeta)=A R_{0}(\zeta)-A R_{0}(\zeta) B T(\zeta),
$$

then $T(\zeta) \mathfrak{S}$ is contained in $\mathscr{D}(B)$, and $T(\zeta)=A R(\zeta)$.

Proof. It is evident from (2.4) and (2.6) that the range of $T(\zeta)$ is contained in $\mathscr{D}(B)$. Put $T=T(\zeta)-A R(\zeta)$. Then for any $f \in \mathfrak{S}$, $T f \in \mathscr{L}(B)$ and $T f=-A R_{0}(\zeta) B T f$. Putting $g=R_{0}(\zeta) B T f$, we have $g=-R_{0}(\zeta) V g$, i.e., $(L-\zeta I) g=0$. Since $\zeta$ is in the resolvent set of $L$, it follows that $g=0$, and hence $T f=-A g=0$ for each $f \in \mathfrak{L}$. This implies $T=T(\zeta)-A R(\zeta)=0$.

Q.E.D.

Let us consider the integral operator $Q_{0}(\kappa)$ with the kernel

6) The factorization of $V$ as given above will be essentially required in $\S 5$ to prove lemmas $5.1 \sim 5$ which play an important role in the following all discussions. A general theory of perturbation by a product operator has been developed by J. Schwartz [10], Kato [13] and Kuroda [16], [17]. 


$$
Q_{0}(x, y ; \kappa)=\frac{a(x) \exp \{i \kappa|x-y|\} b(y)}{4 \pi|x-y|}, \quad \operatorname{Im} \kappa \geq 0 \text {. }
$$

Lemma 2. 2. ${ }^{7)}$ (1) For each fixed $\kappa$ in $\operatorname{Im} \kappa \geq 0, Q_{0}(\kappa)$ is an integral operator of Hilbert-Schmidt type.

(2) $Q_{0}(\kappa)$ is a bounded, and uniformly continuous function of $\kappa$ in $\operatorname{Im} \kappa \geq 0$ in the sense of the operator norm.

Proof. If $a(x)=|q(x)|^{1 / 2}$, then we have, using the Sobolev inequality, ${ }^{8)}$

$$
\begin{aligned}
\int_{E_{3}} \int_{E_{3}}\left|\frac{a(x) b(y)}{4 \pi|x-y|}\right|^{2} d x d y & =(4 \pi)^{-2} \int_{E_{3}} \int_{E_{3}} \frac{|q(x)| \cdot|q(y)|}{|x-y|^{2}} d x d y \\
& \leq \mathrm{const}\left[\int_{E_{3}}|q(x)|^{3 / 2} d x\right]^{4 / 3}<+\infty
\end{aligned}
$$

If $a(x)=(1+|x|)^{(1+\delta) / 2} q(x)$, then we have

$$
\begin{aligned}
\int_{E_{3}} \int_{E_{3}}\left|\frac{a(x) b(y)}{4 \pi|x-y|}\right|^{2} d x d y \leq & (4 \pi)^{-2} \int_{E_{3}}(1+|x|)^{1+\delta}|q(x)|^{2} d x \\
& \times \sup _{x} \int_{E_{3}}|x-y|^{-2}(1+|y|)^{-1-\delta} d y<+\infty .
\end{aligned}
$$

These inequalities show that the kernel $Q_{0}(x, y ; 0)=a(x) \times$ $(4 \pi|x-y|)^{-1} b(y)$ is of Hilbert-Schmidt type for each choice of $a(x)$. Then assertion (1) is obvious since $\left|Q_{0}(x, y ; \kappa)\right| \leq\left|Q_{0}(x, y ; 0)\right|$ for each $\kappa$ in $\operatorname{Im} \kappa \geq 0$. Moreover, we see that $\left\|Q_{0}(\kappa)\right\|$ is bounded by $\left\|Q_{0}(0)\right\|$. Next, given any $\varepsilon>0$, we choose an $R=R(\varepsilon)>0$ such that

$$
\left(\int_{|x|>R} \int_{E_{3}}+\int_{E_{3}} \int_{|y|>R}\right)\left|Q_{0}(x, y ; 0)\right|^{2} d x d y<\varepsilon / 4 \text {. }
$$

Then, by use of the inequality

$$
\left|\exp \{i \kappa|x-y|\}-\exp \left\{i \kappa^{\prime}|x-y|\right\}\right| \leq\left|\kappa-\kappa^{\prime}\right| \cdot|x-y|,
$$

we have for a fixed such $R$

$$
\begin{gathered}
\int_{|x|<R} \int_{i y \mid<R} \frac{|a(x)|^{2}\left|\exp \{i \kappa|x-y|\}-\exp \left\{i \kappa^{\prime}|x-y|\right\}\right|^{2}|b(y)|^{2}}{(4 \pi)^{2}|x-y|^{2}} d x d y \\
\leq(4 \pi)^{-2}\left|\kappa-\kappa^{\prime}\right|^{2} \int_{|x|<R}|a(x)|^{2} d x \int_{|y|<R}|b(y)|^{2} d y<+\infty
\end{gathered}
$$

7) Similar results are already given in [13] and [17].

8) See Mizohata [18]; Lemma 7.1. 
since $a(x)$ and $b(x)$ are both locally square integrable. Taking $\kappa$ and $\kappa^{\prime}$ so near to each other that the right member is bounded by $\varepsilon / 2$, we have finally $\| Q_{0}(\kappa)-\left.Q_{0}\left(\kappa^{\prime}\right)\right|^{2}<\varepsilon$. This proves the uniform continuity of $Q_{0}(\kappa)$ in $\operatorname{Im} \kappa \geq 0$.

Q.E.D.

From (1) of the above lemma, we can apply the Fredholm theory to see that $I+Q_{0}(\kappa)$ has a bounded inverse if and only if the homogeneous equation

$$
\left\{I+Q_{0}(\kappa)\right\} \psi=0, \quad \psi \in \mathfrak{S}
$$

has no non-trivial solution $\psi(\neq 0)$. We call a value $\kappa(\operatorname{Im} \kappa \geq 0)$ a singular point of $Q_{0}(\cdot)$ for which equation (2.8) has non-trivial solutions, and denote by $\Sigma$ the set of all singular points.

We can now state the following results which will be required below.

Theorem 2.1. (1) $\Sigma$ forms in $\operatorname{Im} \kappa \geq 0$ a compact set.

(2) $\Sigma$ is independent of the choice of $a(x): \kappa \neq 0$ belongs to $\Sigma$ if and only if there exists a non-trivial solution $\varphi_{\kappa}(x)$ of the equation

$$
\varphi_{\kappa}(x)=-\int_{E_{3}} \frac{\exp \left\{i_{\kappa}|x-y|\right\}}{4 \pi|x-y|} q(y) \varphi_{\kappa}(y) d y,
$$

which is bounded and satisfies the Sommerfeld radiation condition at infinity:

$$
\varphi_{\kappa}(x)=0\left(|x|^{-1}\right), \quad \lim _{\rho \rightarrow \infty} \int_{\mid x i=\rho}\left|\frac{\partial \varphi_{\kappa}(x)}{\partial|x|}-i \kappa \varphi_{\kappa}(x)\right|^{2} d S=0 .
$$

Hence, when $\operatorname{Im} \kappa>0, \kappa$ belongs to $\Sigma$ if and only if $\mu=\kappa^{2}$ is a discrete eigenvalue of $L$. However, $\mu=\kappa^{2}$ with real $\kappa \in \Sigma$ is not necessarily an eigenvalue of $L$ (cf. Remark 1.1). We denote by $\sigma_{s}(L)$ the set of such values $\mu$. Then this also forms a compact set in $[0, \infty){ }^{97}$

(3) $\left\{I+Q_{0}(\kappa)\right\}^{-1}$ depends continuously on $\kappa$ except for $\kappa \in \Sigma$, and is bounded in the complement in $\operatorname{Im} \kappa \geq 0$ of a neighborhood of $\Sigma$ in the sense of operator norm.

(4) For any $\zeta$ in the resolvent set of $L,\left\{I+Q_{0}(\sqrt{\zeta})\right\}^{-1} A R_{0}(\zeta)$ has the range contained in $\mathscr{D}(B)$, and hence $R(\zeta)$ is represented as

9) In [11], Pavlov investigated the structure of $\sigma_{s}(L)$. 


$$
R(\zeta)=R_{0}(\zeta)-R_{0}(\zeta) B\left\{I+Q_{0}(\sqrt{\zeta})\right\}^{-1} A R_{0}(\zeta)
$$

(5) The set $\Sigma^{*}$ corresponding to the starred operator $Q_{0}^{*}(\kappa)$ with the kernel

$$
Q_{0}^{*}(x, y ; \kappa)=\frac{a \overline{(x)} \exp \left\{i_{\kappa}|x-y|\right\} \overline{b(y)}}{4 \pi|x-y|}
$$

is composed of values $-\bar{\kappa}$ with $\kappa \in \Sigma$, and $R^{*}(\zeta)$ is represented as

$$
R^{*}(\zeta)=R_{0}(\zeta)-R_{0}(\zeta) B^{*}\left\{I+Q_{0}^{*}(\sqrt{\zeta})\right\}^{-1} A^{*} R_{0}(\zeta)
$$

where $A^{*}$ and $B^{*}$ are the multiplicative operators given by $\overline{a(x)}$ and $\overline{b(x)}$, respectively. If we denote by $\sigma_{s}^{*}(L)$ the set of values $\mu=\kappa^{2}$ with real $\kappa \in \Sigma^{*}$, then we have

$$
\sigma_{s}^{*}(L)=\sigma_{s}(L) .
$$

Remark 2.1. If $q(x)$ is assumed to satisfy in addition to (A) the following "smallness" condition

$$
\int_{E_{3}} \int_{E_{3}}\left|q(x) !(4 \pi|x-y|)^{-2}\right| q(y) \mid d x d y<1,
$$

then $I+Q_{0}(\kappa)$ is invertible in $\mathfrak{S}$ for any $\kappa$ in $\operatorname{Im} \kappa \geq 0$, i.e., $\Sigma$ is empty.

Remark 2.2. If $q(x)$ is assumed to satisfy a stronger condition :

$$
q(x) \in L_{\mathrm{loc}}^{2}\left(E_{3}\right), q(x)=0(\exp \{-\delta|x|\}), \delta>0, \quad \text { as } \quad|x| \rightarrow \infty,
$$

then $Q_{0}(\kappa)$ can be continued analytically into the "non-physical sheet" Im $\kappa>-\delta / 2$, preserving the complete continuity (cf. Ladyzenskaja [19] or Kiyama [20]). Therefore we see that no limit point of $\Sigma$ exists in $\operatorname{Im} \kappa \geq 0$, i.e., $\Sigma$ forms a finite set. Consequently, in this case the total multiplicity of the root subspace corresponding to the discrete eigenvalues of $L$ is finite.

Remark 2.3. Let $q(x)$ satisfy in addition to (A) the following "dissipativity" condition :

$$
q_{2}(x)=\operatorname{Im}[q(x)] \leq 0,
$$

and let $q_{1}(x)=\operatorname{Re}[q(x)]$ satisfy the "smallness" condition (2.15). Then $\Sigma$ lies in $\{\kappa ; \operatorname{Re} \kappa<0$ and $\operatorname{Im} \kappa \geq 0\}$. 
Proof. We denote by $V_{1}$ and $A$ the multiplicative operators given by $q_{1}(x)$ and $a(x)=\left(-q_{2}(x)\right)^{1 / 2}$, respectively, i.e., $V=V_{1}-i A^{2}$. Let $\kappa=\mu+i \tau$ be in $\Sigma$. Then (2.9) has a non-trivial solution satisfying (2.10). First assume $\tau>0$. Then (2.9) can be written as $\varphi_{\kappa}(x)+\left[R_{0}\left(\kappa^{2}\right) V \varphi_{\kappa}\right](x)=0\left(\varphi_{\kappa} \in \mathfrak{L}\right)$. Multiplying by $\overline{q(x) \varphi_{\kappa}(x)}$ both sides and integrating over $E_{3}$, we get

$$
\left(\varphi_{\kappa}, V \varphi_{\kappa}\right)+\left(R_{0}\left(\kappa^{2}\right) V \varphi_{\kappa}, V \varphi_{k}\right)=0 .
$$

This implies that the imaginary part of the left side also equals zero :

$$
-|| A \varphi_{\kappa} \|^{2}-\int_{0}^{\infty} \frac{2 \mu \tau}{\left(\lambda-\mu^{2}+\tau^{2}\right)^{2}+(2 \mu \tau)^{2}} d\left(E_{0, \lambda} V \varphi_{\kappa}, V \varphi_{\kappa}\right)=0 .
$$

Here $E_{0, \lambda}$ is the resolution of the identity of the selfadjoint operator $L_{0}$. This equality shows that $a(x) \varphi_{\kappa}(x) \equiv 0$ if $\mu \geq 0$. Hence $\varphi_{\mathrm{k}}+R_{0}\left(\kappa^{2}\right) V_{1} \varphi_{\mathrm{k}}=0$ if $\mu \geq 0$ and $\tau>0$. However, since $L_{1}=L_{0}+V_{1}$ is selfadjoint, this implies that $\varphi_{k}(x) \equiv 0$. Next, if $\tau=0$, i.e., $\kappa=\mu$, then non-trivial solutions of (2.9) are no longer in $\mathfrak{S}$. However, we see from (2.10) that $a(x) \varphi_{k}(x)$ is in $\mathfrak{S}$. Hence we can follow a way similar to that given above to get

$$
-|| a \varphi_{\kappa} \|^{2}-\pi \frac{\mu}{|\mu|}\left[\frac{d}{d \lambda}\left(E_{0, \lambda} q \varphi_{\kappa}, q \varphi_{\kappa}\right)\right]_{\lambda=\mu^{2}}=0,
$$

which also implies $a(x) \varphi_{\kappa}(x) \equiv 0$ if $\mu \geq 0$. On the other hand, by Remark 2.1, we see that the operator function $Q_{0}(x)$ corresponding to $L_{1}=L_{0}+V_{1}$ has no singular point. Hence we get $\varphi_{\kappa}(x) \equiv 0$, and the proof of Remark 2.3 is completed.

Q.E. D.

\section{§ 3. Proof of Theorem 2.1.}

Lemma 3. 1. Suppose that $0<\alpha<3, \alpha+\beta>3$, and $\beta \neq 3$. Then we have

$$
\begin{aligned}
\int_{E_{3}}|x-y|^{-\alpha}(1+|y|)^{-\beta} d y & \leq \text { const }(1+|x|)^{-\gamma}, \\
\gamma & =\min (\alpha, \alpha+\beta-3) .
\end{aligned}
$$

Proof. It is evident that the integral is bounded in $x \in E_{3}$. So 
we have only to show (3.1) in the case where $|x| \geq 1$. If $\beta<3$, putting $y /|x|=z$, we have

$$
\begin{gathered}
\int_{E_{3}}|x-y|^{-\infty}(1+|y|)^{-\beta} d y=|x|^{-\alpha-\beta+3} \int_{E_{3}}\left|\frac{x}{|x|}-z\right|^{-\infty}|z|^{-\beta} d z \\
\leq \text { const }|x|^{-\infty-\beta+3} .
\end{gathered}
$$

If $\beta>3$, taking account of the inequality

(3.2) $|x-y|^{-\infty}(1+|y|)^{-\infty} \leq$ const $(1+|x|)^{-\infty}\left\{|x-y|^{-\infty}+(1+|y|)^{-\infty}\right\}$,

we get

$$
\begin{aligned}
& \int_{E}|x-y|^{-\infty}(1+|y|)^{-\beta} d y \\
\leq & \operatorname{const}(1+|x|)^{-\infty} \int_{E_{3}}\left\{|x-y|^{-\infty}(1+|y|)^{-\beta+\alpha}+(1+|y|)^{-\beta}\right\} d y \\
\leq & \operatorname{const}(1+|x|)^{-\alpha} .
\end{aligned}
$$

These prove inequality (3.1).

If we denote by $Q_{0}^{(n)}(x, y ; \kappa)$ the kernel of $Q_{0}(\kappa)^{n}(n=1,2, \cdots)$, then it is represented as follows:

$$
Q_{0}^{(n)}(x, y ; \kappa)=a(x) P_{0}^{(n)}(x, y ; \kappa) b(y),
$$

where $P_{0}^{(1)}(x, y ; \kappa)=(4 \pi|x-y|)^{-1} \exp \left\{i_{\kappa}|x-y|\right\}$, and

$$
P_{0}^{(n)}(x, y ; \kappa)=\int_{E_{3}} \frac{\exp \left\{i_{\kappa}|x-z|\right\}}{4 \pi|x-z|} q(z) P_{0}^{(n-1)}(z, y ; \kappa) d z .
$$

Lemma 3.2. Let $\delta^{\prime}$ be an arbitrary constant such that $0<\delta^{\prime}$ $<\min (1, \delta)$. Then we have

$$
\begin{aligned}
& \left|P_{0}^{(2)}(x, y ; \kappa)\right| \leq \mathrm{const}\|q\|_{1+\delta}\left\{(1+|x|)^{-1}+(1+|x|)^{-\left(1+\delta^{\prime}\right) / 2}|x-y|^{-1 / 2}\right\}, \\
& \left|P_{0}^{(2)}(x, y ; \kappa)\right| \leq \mathrm{const} \|\left. q\right|_{1+\delta}\left\{(1+|y|)^{-1}+(1+|y|)^{-\left(1+\delta^{\prime}\right) / 2}|x-y|^{-1 / 2}\right\},
\end{aligned}
$$

where the "const" is independent of $\kappa$ and

$$
\|q\|_{1+\delta}=\left[\int_{E_{3}}(1+|x|)^{1+\delta}|q(x)|^{2} d x\right]^{1 / 2} .
$$

Proof. Since $P_{0}^{(2)}(x, y ; \kappa)$ is symmetric in $x$ and $y$, we have only to show the first inequality. Without loss of generality, we can assume $\delta<1$. By use of the Schwarz inequality, it follows that 


$$
\begin{aligned}
\left|P_{0}^{(2)}(x, y ; \kappa)\right| & \leq(4 \pi)^{-2} \int_{E_{3}}|x-z|^{-1}|q(z)| \cdot|z-y|^{-2} d z \\
& \leq(4 \pi)^{-2}|| q \|_{1+\delta}\left[\int_{E_{3}}|x-z|^{-2}(1+|z|)^{-1-\delta}|z-y|^{-2} d z\right]^{1 / 2} .
\end{aligned}
$$

We use (3.2) to obtain

$$
\begin{aligned}
& |x-z|^{-2}(1+|z|)^{-1-\delta}|z-y|^{-2} \\
\leq & \text { const }(1+|x|)^{-1-\delta}\left\{|x-z|^{-2}+|x-z|^{-1+\delta}(1+|z|)^{-1-\delta}\right\}|z-y|^{-2} \\
\leq & \text { const }(1+|x|)^{-1-\delta}|x-z|^{-2}|z-y|^{-2} \\
& + \text { const }(1+|x|)^{-2}\left\{|x-z|^{-1+\delta}(1+|z|)^{-2 \delta}+(1+|z|)^{-1-\delta}\right\}|z-y|^{-2} .
\end{aligned}
$$

It is not difficult to see that $\int_{E_{3}}|x-z|^{-2}|z-y|^{-2} d z \leq$ const $|x-y|^{-1}$. On the other hand, since $\delta<1$, we have

$$
\int_{E_{3}}\left\{|x-z|^{-1+\delta}(1+|z|)^{-2 \delta}+(1+|z|)^{-1-\delta}\right\}|z-y|^{-2} d z \leq \text { const }<+\infty .
$$

Summarizing these inequalities, we finally get the inequality

$$
\begin{aligned}
& \int_{E_{3}}|x-z|^{-2}(1+|z|)^{-1-\delta}|z-y|^{-2} d z \\
\leq & \text { const }(1+|x|)^{-1-\delta}|x-y|^{-1}+\operatorname{const}(1+|x|)^{-2},
\end{aligned}
$$

which proves the first inequality of (3.5). Q. E. D.

Using estimates (3.5), we can prove the following two lemmas.

Lemma 3. 3. For any $f(x) \in \mathfrak{S}$ we have

$$
\left|\left[Q_{0}(\kappa)^{4} f\right](x)\right| \leq \mathrm{const}|a(x)|(1+|x|)^{-\gamma}|| f||,
$$

where $\gamma=1 / 2$ when $a(x)=a_{1}(x)$, and $0<\gamma<\min (1 / 2, \delta / 2)$ when $a(x)=a_{2}(x)$. Hence $Q_{0}(\kappa)^{4} f \in \mathscr{D}(B)$. Moreover, if a positive integer $n$ is taken sufficiently large, then we have

$$
\left|\left[Q_{0}(\kappa)^{n} f\right](x)\right| \leq \text { const }|a(x)|(1+|x|)^{-1}|| f||
$$

for any choice of $a(x)$. The "const" in the above inequalities is independent of $\kappa$.

Proof. $P_{0}^{(2)}(x, y ; \kappa)$ being estimated by (3.5), we can make use of the Fubini theorem to represent (3.4) for $n=4$ as follows :

$$
P_{0}^{(4)}(x, y ; \kappa)=\int_{E_{3}} P_{0}^{(2)}(x, z ; \kappa) q(z) P_{0}^{(2)}(z, y ; \kappa) d z .
$$


Let us estimate this integral with respect to $x$. By virtue of (3.5), we have

$$
\begin{aligned}
& \left|P^{(4)}(x, y ; \kappa)\right| \\
\leq & \operatorname{const}(1+|x|)^{-1} \int_{E_{3}}|q(z)|\left\{(1+|z|)^{-1}+(1+|z|)^{-\left(1+\delta^{\prime}\right) / 2}|z-y|^{-1 / 2}\right\} d z \\
& +\operatorname{const}(1+|x|)^{-\left(1+\delta^{\prime}\right) / 2} \int_{E_{3}}|x-z|^{-1 / 2}|q(z)|\left\{(1+|z|)^{-1}+\right. \\
& \left.+(1+|z|)^{-\left(1+\delta^{\prime}\right) / 2}|z-y|^{-1 / 2}\right\} d z .
\end{aligned}
$$

By use of the Schwarz inequality, we see that the first integral in the right member is bounded in $y$. The second integral is estimated as $\int_{E_{3}}|x-z|^{-1 / 2}|q(z)|(1+|z|)^{-1} d z \leq\left[\int_{E_{3}}|x-z|^{-1}(1+|z|)^{-3-\delta} d z\right]^{1 / 2}\|q\|_{1+\delta}$

$$
\begin{aligned}
& \leq \text { const }(1+|x|)^{-1 / 2}, \\
& \int_{E_{3}}|x-z|^{-1 / 2}|q(z)|(1+|z|)^{-\left(1+\delta^{\prime} / 2 / 2\right.}|z-y|^{-1 / 2} d z
\end{aligned}
$$

$\leq\left[\int_{E_{3}}|x-z|^{-2}(1+|z|)^{-3\left(1+\delta^{\prime}\right)} d z\right]^{1 / 4}\left[\int_{E_{3}}|z-y|^{-2}(1+|z|)^{-1-\delta^{\prime}} d z\right]^{1 / 4}\|q\|_{1+\delta^{\prime}}$ $\leq$ const $(1+|x|)^{-1 / 2}$,

with the aid of Lemma 3.1. Thus we get

$$
\begin{aligned}
\left|P_{0}^{(4)}(x, y ; \kappa)\right| & \leq \mathrm{const}(1+|x|)^{-1}+\text { const }(1+|x|)^{-\left(2-\delta^{\prime}\right) / 2} \\
& \leq \text { const }(1+|x|)^{-1} .
\end{aligned}
$$

This inequality is also valid with $(1+|x|)^{-1}$ replaced by $(1+|y|)^{-1}$. So we have

$$
\left|P_{0}^{(4)}(x, y ; \kappa)\right| \leq \text { const }(1+|x|)^{-\gamma}(1+|y|)^{-1, \gamma}
$$

for an arbitrary constant $\gamma$ such that $0 \leq \gamma \leq 1$. If we choose $\gamma$ as given in the lemma, then both $a(x)(1+|x|)^{-\gamma}$ and $b(x)(1+|x|)^{-1+\gamma}$ are in $L^{2}\left(E_{3}\right)$ for each choice of $a(x)$. Hence we have from (3.3)

$$
\begin{aligned}
\left|\left[Q_{0}(\kappa)^{4} f\right](x)\right| & \leq \text { const }|a(x)|(1+|x|)^{-\gamma}\left|\int_{E_{3}} b(y)(1+|y|)^{-1+\gamma} f(y) d y\right| \\
& \leq \text { const }|a(x)|(1+|x|)^{-\gamma}|| f|| .
\end{aligned}
$$

This proves the first half of the lemma. Inequality (3.9) is proved by iteration with the aid of the Schwarz inequality and Lemma 3.1.

Q. E. D. 
Lemma 3. 4. $Q_{0}(\kappa)^{2}$ vanishes as $|\kappa| \rightarrow \infty$, i.e., given any $\varepsilon>0$ there exists a constant $\kappa_{0}(\varepsilon)>0$ such that

$$
|| Q_{0}(\kappa)^{2} \mid:<\varepsilon, \quad \text { if } \quad|\kappa| \geq \kappa_{0}(\varepsilon) \text {. }
$$

Proof. We choose $q_{0}(x) \in C_{0}^{\infty}\left(E_{3}\right)$, and put $\widetilde{q}(x)=q(x)-q_{0}(x)$. Obviously $\widetilde{q}(x)$ satisfies condition (A). Since $C_{0}^{\infty}\left(E_{2}\right)$ is dense in $\mathfrak{g}$, we can choose $q_{0}(x) \in C_{0}^{\infty}\left(E_{2}\right)$ such that $\|\widetilde{q}\|_{1+\delta}$ becomes as small as we wish. We write

$$
\begin{aligned}
& P_{0}^{(2)}(x, y ; \kappa)=P_{01}^{(2)}(x, y ; \kappa)+P_{02}^{(2)}(x, y ; \kappa) \\
= & \int_{E_{3}} \frac{\exp \{i \kappa(|x-z|+|z-y|)\}}{(4 \pi)^{2}|x-z| \cdot|z-y|} q_{0}(z) d z \\
& \quad+\int_{E_{3}} \frac{\exp \left\{i_{\kappa}(|x-z|+|z-y|)\right\}}{(4 \pi)^{2}|x-z| \cdot|z-y|} \widetilde{q}(z) d z .
\end{aligned}
$$

It is known by a Lemma of Faddeev [4] that ${ }^{10)}$

$$
\left|P_{01}^{(2)}(x, y ; \kappa)\right| \leq \text { const }(1+|\kappa|)^{-1}(1+|x-y|)^{-1},
$$

where the "const" depends only on the choice of $q_{0}(x)$. Using this we have

$$
\int_{E_{3}} \int_{E_{3}}\left|a(x) P_{01}^{(2)}(x, y ; \kappa) b(y)\right|^{2} d x d y \leq(1+|\kappa|)^{-2} .
$$

On the other hand, choosing $\gamma(\gamma \leq 1 / 2)$ as given in Lemma 3.3, we have

$$
\begin{aligned}
& \left|a(x) P_{02}^{(2)}(x, y ; \kappa) b(y)\right|^{2} \\
= & |a(x)|^{2}\left|P_{02}^{(2)}(x, y ; \kappa)\right|^{2 \gamma}\left|P_{02}^{(2)}(x, y ; \kappa)\right|^{2(1-\gamma)}|b(y)|^{2} \\
\leq & \mathrm{const}\|\widetilde{q}\|_{1+\delta}^{2} 2\left\{|a(x)|^{2}(1+|x|)^{-2 \gamma}|b(y)|^{2}(1+|y|)^{-2,1-\gamma)}\right. \\
& \left.+|a(x)|^{2}(1+|x|)^{-(1+\delta \prime) \gamma}|x-y|^{-1}|b(y)|^{2}(1+|y|)^{-(1+\delta /)(1-\gamma)}\right\}
\end{aligned}
$$

by (3.5). Taking into account that $a(x)(1+|x|)^{-\gamma}, b(x)(1+|x|)^{-1+\gamma} \in$ $L^{2}\left(E_{3}\right)$ and $|a(x)| \cdot|x-y|^{-1}|b(y)| \in L^{2}\left(E_{3} \times E_{3}\right)$, we then have

$$
\int_{E 3} \int_{E_{3}}\left|a(x) P_{02}^{(2)}(x, y ; \kappa) b(y)\right|^{2} d x d y \leq \mathrm{const}\|\widetilde{q}\|_{1_{\top \delta}}^{2},
$$

with the "const" independent of $\kappa$. (3.11) and (3.12) imply inequality (3.10).

10) See also Ladyzenskaja [19]. 
We can now prove Theorem 2.1.

Q.E.D.

Proof of Theorem 2.1. (1) By virtue of (3.10), the bounded inverse of $I+Q_{0}(\kappa)$ can be constructed as

$$
\left\{I+Q_{0}(\kappa)\right\}^{-1}=\left\{I-Q_{0}(\kappa)\right\}\left\{I+Q_{0}(\kappa)^{2}\right\}^{-1}=\left\{I-Q_{0}(\kappa)\right\} \sum_{n=0}^{\infty} Q_{0}(\kappa)^{2 n}
$$

for each $|\kappa| \geq \kappa_{0}(1)$. Hence $\Sigma$ forms a bounded set contained in the disk $\left\{\kappa ;|\kappa|<\kappa_{0}(1)\right\}$. The closedness of $\Sigma$ follows from the fact that $Q_{0}(\kappa)$ is a completely continuous operator which depends continuously on $\kappa$ (cf. Povzner [2]; II, Theorem 4).

(2) Put $\psi_{\kappa}(x)=a(x) \varphi_{\kappa}(x)$ for any non-trivial bounded solution $\varphi_{k}(x)$ of equation (2.9) satisfying (2.10). Then $\psi_{k}(x) \neq 0$, and $\in \mathcal{E}$ by (2.10). Multiplying both sides of (2.9) by $a(x)$, we get $\psi_{k}=-Q_{0}(\kappa) \psi_{\kappa}$, which shows $\kappa \in \Sigma$.

Conversely, let $\kappa \neq 0$ be in $\Sigma$, and $\psi_{\kappa}(x) \in \mathfrak{S}$ be a non-trivial solution of equation (2.8). Then, since

$$
\psi_{\kappa}=-Q_{0}(\kappa) \psi_{\kappa}=\cdots=(-1)^{n} Q_{0}(\kappa)^{n} \psi_{\kappa}=\cdots,
$$

we have from Lemma 3.3

$$
\left|\psi_{\kappa}(x)\right| \leq \text { const }|a(x)|(1+|x|)^{-1}|| \psi_{\kappa}|| .
$$

Put

$$
\varphi_{\kappa}(x)=-\int_{E_{3}} \frac{\exp \left\{i_{\kappa}|x-y|\right\}}{4 \pi|x-y|} b(y) \psi_{\kappa}(y) d y .
$$

Then we have $\psi_{\kappa}(x)=a(x) \varphi_{\kappa}(x)$, and hence $\varphi_{\kappa}(x) \neq 0$. With the aid of Lemma 3.1, it follows from (3.14) and (3.15) that

$$
\begin{aligned}
\left|\varphi_{\kappa}(x)\right| & \leq \text { const }\|q\|_{1+\delta}\left[\int_{E_{3}}(1+|y|)^{-3-\delta}|x-y|^{-2} d y\right]^{1 / 2} \\
& \leq \text { const }(1+|x|)^{-1},
\end{aligned}
$$

i.e., $\varphi_{k}(x)$ is bounded and behaves like $0\left(|x|^{-1}\right)$ at infinity. To proceed with the proof, we use the following lemma due to $\dot{E}_{\text {idus }}$ ([9]; Lemma 4).

Lemma 3.5. Let $f(x)$ be a function such that

$$
(1+|x|)^{(3+\delta) / 2} f(x) \in L^{2}\left(E_{3}\right) .
$$


Then the function $g(x)=\int_{E_{3}} \frac{\exp \{i \kappa|x-y|\}}{4 \pi|x-y|} f(y) d y$ satisfies the inequality

(3. 17) $\int_{|x|=\rho}\left|\frac{\partial g(x)}{\partial|x|}-i \kappa g(x)\right|^{2} d S \leq$ const $\rho^{-\gamma}$, where $\gamma=\min (1,2 \delta /(2+\delta))$.

Now we have from (3.14)

$$
\left|(1+|\kappa|)^{(3+\delta) / 2} b(x) \psi_{\kappa}(x)\right| \leq \mathrm{const}(1+|x|)^{(1+\delta) / 2}|q(x)| \in L^{2}\left(E_{3}\right) .
$$

Hence, from (3.15) and the above lemma, $\varphi_{\kappa}(x)$ is shown to satisfy the radiation condition (2.10). Substituting $\psi_{\kappa}(x)=a(x) \varphi_{\kappa}(x)$ in (3.15), we conclude that $\varphi_{\kappa}(x)$ is a non-trivial solution of (2.9), (2.10).

Finally, in the case $\operatorname{Im} \kappa>0$, every bounded solution of (2.9) belongs to $\mathscr{D}\left(L_{0}\right)$, and hence we can rewrite (2.9) as $\varphi_{\kappa}=R_{0}\left(\kappa^{2}\right) V \varphi_{\kappa}$. This implies that $\kappa^{2}$ is an eigenvalue of $L$ if and only if (29) has a non-trivial bounded solution. Consequently $\kappa \in \Sigma(\operatorname{Im} \kappa>0)$ if and only if $\kappa^{2}$ is an eigenvalue of $L$.

(3) Since $Q_{0}(\kappa)$ depends continuously on $\kappa$, and $\Sigma$ is closed, given any $\kappa \notin \Sigma$ and $0<\varepsilon<1$, there exists a constant $\delta=\delta(\kappa, \varepsilon)>0$ such that

$$
\|\left\{Q_{0}(\kappa)-Q_{0}\left(\kappa^{\prime}\right)\right\}\left\{I+Q_{0}(\kappa)\right\}^{-1} \mid: \leq \varepsilon / 2, \quad \text { if } \quad\left|\kappa^{\prime}-\kappa\right|<\delta .
$$

For each such $\kappa^{\prime},\left\{I+Q_{0}\left(\kappa^{\prime}\right)\right\}^{-1}$ exists and admits the Neumann series expansion :

$$
\begin{aligned}
& \left\{I+Q_{0}\left(\kappa^{\prime}\right)\right\}^{-1}-\left\{I+Q_{0}(\kappa)\right\}^{-1} \\
= & \left\{I+Q_{0}(\kappa)\right\}^{-1} \sum_{n=1}^{\infty}\left[\left\{Q_{0}(\kappa)-Q_{0}\left(\kappa^{\prime}\right)\right\}\left\{I+Q_{0}\left(\kappa^{\prime}\right)\right\}^{-1}\right]^{n} .
\end{aligned}
$$

Thus we have

$$
\left\|\left\{I+Q_{0}\left(\kappa^{\prime}\right)\right\}^{-1}-\left\{I+Q_{0}(\kappa)\right\}^{-1}\right\| \leq \varepsilon\left\|^{\prime}\left\{I+Q_{0}(\kappa)\right\}^{-1}\right\|, \quad \text { if } \quad\left|\kappa^{\prime}-\kappa\right|<\delta .
$$

This implies that $\left\{I+Q_{0}(\kappa)\right\}^{-1}$ is a continuous function of $\kappa$ except for $\kappa \in \Sigma$. The boundedness of $\left\{I+Q_{0}(\kappa)\right\}^{-1}$ in the complement in $\operatorname{Im} \kappa \geq 0$ of a neighborhood of $\Sigma$ follows from (3.13) and the continuity of $\left\{I+Q_{0}(\kappa)\right\}^{-1}$.

(4) Let $\zeta=\kappa^{2}(\operatorname{Im} \kappa>0)$ be in the resolvent set of $L$, and put

$$
T(\kappa)=\left\{I+Q_{0}(\kappa)\right\}^{-1} A R_{0}(\zeta) .
$$

Then we have 


$$
T(\zeta)=\sum_{n=0}^{3}(-1)^{n} Q_{0}(\kappa)^{n} A R_{0}(\zeta)+Q_{0}(\kappa)^{4} T(\zeta)
$$

It is proved in Lemma 3.3 that the range of $Q_{0}(\kappa)^{4}$ is included in $\mathscr{D}(B)$. On the other hand, since also $A R_{0}(\zeta) \mathfrak{S} \subset \mathscr{D}(B)$ by (2.4), we have

$$
\begin{gathered}
Q_{0}(\kappa)^{n} A R_{0}(\zeta)=Q_{0}(\kappa)^{n-1} A R_{0}(\zeta) V R_{0}(\zeta)=\cdots=A R_{0}(\zeta)\left[V R_{0}(\zeta)\right]^{n} \\
n=0,1,2, \cdots
\end{gathered}
$$

Thus we see that $T(\zeta)$ has the range included in $\mathscr{D}(B)$, and then gives a solution of equation (2.6). Consequently we have from Lemma 2.1 $T(\zeta)=A R(\zeta)$. Substituting (3.18) in the second member of (2.5), we have finally (2.11).

(5) We have only to prove the assertion that $\kappa \in \Sigma$ if and only if $-\bar{\kappa} \in \Sigma^{*}$, which is obvious since we have for any $f \in \mathfrak{N}$

$$
\overline{\left[Q_{0}(\kappa) f\right](x)}=\int_{E_{3}} \frac{\overline{a(x)} \exp \{-i \bar{\kappa}|x-y|\} \overline{b(y)}}{4 \pi|x-y|} \overline{f(y)} d y=\left[Q_{0}^{*}(-\bar{\kappa}) \bar{f}\right](x) .
$$

\section{Eigenfunction Expansions}

\section{$\$ 4$. Distorted plane waves.}

Let us consider the conjugate Fourier transform $r(x, k ; \kappa)$ of the resolvent kernel $R(x, y, \kappa)$ with defining equation

$$
r(x, k ; \kappa)=(2 \pi)^{-3 / 2} \int_{E_{3}} R(x, y ; \kappa) \exp \{i k \cdot y\} d y^{11)}
$$

for each $x$ and $\operatorname{Im} \kappa>0(\kappa \notin \Sigma)$, noting that $R(x, \cdot ; \kappa) \in L^{1}\left(E_{3}\right) \cap L^{2}\left(E_{3}\right)$ (see Theorem 1.2). Since

$$
\int_{E_{3}} \frac{\exp \{i \kappa|x-y|\}}{4 \pi|x-y|} \exp \{i k \cdot y\} d y=\frac{\exp \{i k \cdot x\}}{|k|^{2}-\kappa^{2}}
$$

and $\exp \left\{i_{\kappa}|x-y|\right\}|x-y|^{-1} q(y) \in L^{1}\left(E_{3}\right)$ as function of $y$, we get from (1. 12)

11) $k \cdot x$ denotes the scalar product of $k$ and $x$. 


$$
\begin{aligned}
r(x, k ; \kappa)=(2 \pi)^{-3 / 2} \frac{\exp \{i k \cdot x\}}{|k|^{2}-\kappa^{2}}-\int_{E_{3}} \frac{\exp \{i \kappa|x-y|\}}{4 \pi|x-y|} \\
\times q(y) r(y, k ; \kappa) d y
\end{aligned}
$$

where we have used the Fubini theorem to interchange the integration order in the last integral.

Now we fix the factorization $q(x)=b(x) a(x)$ by choosing $a(x)=$ $a_{2}(x)=(1+|x|)^{(1+\delta) / 2} q(x) \in L^{2}\left(E_{3}\right)$. Then it follows from Lemma 1.1 that $A R(\zeta)=\left\{I+Q_{0}(\kappa)\right\}^{-1} A R_{0}(\zeta), \zeta=\kappa^{2}$, is an integral operator of Hilbert-Schmidt type. So the Parseval equality shows that $a_{2}(x) r(x, k ; \kappa) \in L^{2}\left(E_{3} \times E_{3}\right)$ for each $\operatorname{Im} \kappa>0 \quad(\kappa \notin \Sigma)$. Putting

$$
\psi(x, k ; \kappa)=(2 \pi)^{3 / 2}\left(|k|^{2}-\kappa^{2}\right) a_{2}(x) r(x, k ; \kappa),
$$

we have $\psi(\cdot, k ; \kappa) \in L^{2}\left(E_{3}\right)$, and from (4.3)

$$
\begin{aligned}
\psi(\cdot, k ; \kappa) & =\psi_{0}(\cdot, k)-Q_{0}(\kappa) \psi(\cdot, k ; \kappa) ; \\
\psi_{0}(x, k) & =a_{2}(x) \exp \{i k \cdot x\} .
\end{aligned}
$$

As discussed in $\S 2$, this equation has a unique solution in $\mathfrak{S}$ for each $k$ and $\operatorname{Im} \kappa \geq 0(\kappa \notin \Sigma)$. Thus we can extend the definition of $\psi(x, k ; \kappa)$ to the case where $\operatorname{Im} \kappa=0(\kappa \notin \Sigma)$ by the solution of (4.5).

Lemma 4.1. $\psi(\cdot, k ; \kappa)$ is an $\mathfrak{S}$-valued bounded, continuous function of $k \in E_{3}$ and $\kappa \in \rho_{\Sigma}$, where $\rho_{\Sigma}$ is the complewent in $\operatorname{Im} \kappa \geq 0$ of a neighborhood of $\Sigma$.

Proof. Since we have $\left\|\psi_{0}(\cdot, k)\right\| \leq \| a_{2} \mid$ and

$$
\left|! \psi_{0}(\cdot, k)-\psi_{0}\left(\cdot, k^{\prime}\right)\right|^{2} \leq 2 \int_{|x|>R}\left|a_{2}(x)\right|^{2} d x+\left|k-k^{\prime}\right|^{2} \int_{|x|<R}\left|a_{2}(x)\right|^{2}|x|^{2} d x,
$$

it follows that $\psi_{0}(\cdot, k)$ is an $\mathfrak{S}_{2}$-valued bounded and uniformly continuous function of $k$. Hence the lemma is proved by (3) of Theorem 2. 1 .

Q. E.D.

We put

$$
\begin{aligned}
& \varphi(x, k ; \kappa)=\exp \{i k \cdot x\}+v(x, k ; \kappa) \\
& v(x, k ; \kappa)=-\int_{E_{3}} \frac{\exp \{i \kappa|x-y|\}}{4 \pi|x-y|} b_{2}(y) \psi(y, k ; \kappa) d y .
\end{aligned}
$$

Then we have the 
Lemma 4. 2. (1) $v(x, k ; \kappa)$ is bounded, continuous in $x \in E_{3}, k \in E_{3}$, and $\kappa \in \rho_{\Sigma}$, where $\rho_{\Sigma}$ is as given in Lemma 4.1. Moreover, we have

$$
|v(x, k ; \kappa)| \leq \mathrm{const}(1+|x|)^{-\delta^{\prime} / 2}, \quad 0<\delta^{\prime}<\min (2, \delta),
$$

where the "const" depends only on the choice of $\rho_{\Sigma}$.

(2) $\varphi(x, k ; \kappa)$ is a unique bounded solution of the integral equation (4. 9) $\varphi(x, k ; \kappa)=\exp \{i k \cdot x\}-\int_{E_{3}} \frac{\exp \{i \kappa|x-y|\}}{4 \pi|x-y|} q(y) \varphi(y, k ; \kappa) d y, \kappa \notin \Sigma$.

Proof. (1) Noting $b_{2}(x)=(1+|x|)^{-(1+\delta) / 2}$, we have

$$
|v(x, k ; \kappa)| \leq(4 \pi)^{-1}\left[\int_{E_{3}}|x-y|^{-2}(1+|y|)^{-1-\delta} d y\right]^{1 / 2}\|\psi(\cdot, k ; \kappa)\| .
$$

Hence we get (4.8) by Lemma 3.1. By Lemma 4.1 and the inequality

$$
\begin{aligned}
& \int_{E_{3}} \frac{\left|\exp \{i \kappa|x-y|\}-\exp \left\{i \kappa^{\prime}|x-y|\right\}\right|^{2}}{|x-y|^{2}}\left|b_{2}(y)\right|^{2} d y \\
\leq & \left|\kappa-\kappa^{\prime}\right|^{2} \int_{|y|<R}(1+|y|)^{-1-\delta} d y+2 \int_{|y|>R}|x-y|^{-2}(1+|y|)^{-1-\delta} d y,
\end{aligned}
$$

we see that $v(x, k ; \kappa)$ is uniformly continuous in $k, \kappa$. The continuity in $x$ follows from the inequality

$$
\begin{aligned}
& \int_{E_{3}}\left|\frac{\exp \left\{i_{\kappa}|x-y|\right\}}{|x-y|}-\frac{\exp \left\{i_{\kappa}\left|x^{\prime}-y\right|\right\}}{\left|x^{\prime}-y\right|}\right|^{2}\left|b_{2}(y)\right|^{2} d y \\
& \leq|\kappa|^{2}\left|x-x^{\prime}\right|^{2} \int_{E_{3}}|x-y|^{-2}(1+|y|)^{-1-\delta} d y+ \\
& +\left|x-x^{\prime}\right|^{2} \int_{E_{3}}|x-y|^{-2}\left|x^{\prime}-y\right|^{-2}(1+|y|)^{-1-\delta} d y
\end{aligned}
$$

in virtue of (3.7).

(2) Multiplying (4.6) by $a_{2}(x)$, we see from (4.5) that $a_{2}(x) \varphi(x, k ; \kappa)=\psi(x, k ; \kappa)$. Substituting this in (4.7), we find that $\varphi(x, k ; \kappa)$ is a solution of (4.9). Conversely, let $\widetilde{\mathcal{P}}$ be a bounded solution of (4.9). Then, since $\widetilde{\psi}=a_{2}(x) \widetilde{\rho}$ satisfies (4.5), and the solution of (4.5) is unique if $\kappa \notin \Sigma$, we find that $\widetilde{\psi}=\psi$, and hence that $\widetilde{\rho}=\varphi$.

Q.E.D.

Now from (4.3), (4.4) and (4.6) it follows that (4. 10) $\quad r(x, k ; \kappa)=(2 \pi)^{-3 / 2}\left(|k|^{2}-\kappa^{2}\right)^{-1} \varphi(x, k ; \kappa)$, if $\operatorname{Im} \kappa>0(\kappa \notin \Sigma)$. 
Similarly, we can construct the function $\phi^{*}(x, k ; \kappa)=\exp \{i k \cdot x\}+$ $v^{*}(x, k ; \kappa), \operatorname{Im} \kappa \geq 0(-\bar{\kappa} \notin \Sigma$; cf. (5) of Theorem 2.1) as a unique bounded solution of (4.9) with $q(x)$ replaced by $\overline{q(x)}$. Then we have also the following representation of $r^{*}(x, k ; \kappa)$ :

$$
\begin{aligned}
& r^{*}(x, k ; \kappa)=(2 \pi)^{-3 / 2}\left(|k|^{2}-\kappa^{2}\right)^{-1} \varphi^{*}(x, k ; \kappa), \\
& \text { if } \quad \operatorname{Im} \kappa>0(-\bar{\kappa} \notin \Sigma),
\end{aligned}
$$

where $r^{*}(x, k ; x)$ is the conjugate Fourier transform of $R^{*}(x, y ; \kappa)$ :

$$
r^{*}(x, k ; \kappa)=(2 \pi)^{-3 / 2} \int_{E_{3}} R^{*}(x, y ; \kappa) \exp \{i k \cdot y\} d y .
$$

Finally we put

(4.13) $\varphi_{ \pm}(x, k)=\exp \{i k \cdot x\}+v_{ \pm}(x, k) \equiv \varphi(x, k ; \mp|k|), \quad \mp|k| \notin \Sigma$,

(4.14) $\phi_{ \pm}^{*}(x, k)=\exp \{i k \cdot x\}+v_{ \pm}^{*}(x, k) \equiv \varphi^{*}(x, k ; \mp|k|), \quad \pm|k| \notin \Sigma$.

Then we arrive at the following

Theorem 4.1. ${ }^{12)} \quad \varphi_{\tau}(x, k)$ and $\varphi_{-}(x, k)$ are the so-called distorted plane waves, having the forms (plane wave)+(incoming wave) and (plane wave)+(outgoing wave), respectively, and satisfying the Lippmann-Schwinger equation

$$
\varphi_{ \pm}(x, k)=\exp \{i k \cdot x\}-\int_{E_{3}} \frac{\exp \{\mp i|k| \cdot|x-y|\}}{4 \pi|x-y|} q(y) \varphi_{ \pm}(y, k) d y,
$$

that is, satisfying the Schrödinger

$$
-\Delta \varphi_{ \pm}+q(x) \phi_{ \pm}=|k|^{2} \varphi_{ \pm}
$$

in the distribution sense. $\phi_{+}^{*}(x, k)$ and $\varphi_{-}^{*}(x, k)$ are the corresponding adjoint waves satisfying (4.15) and (4.16) with $q(x)$ replaced by $\overline{q(x)}$.

Remark 4.1. It should be noted that under our assumption (A) on $q(x)$, the incoming or outgoing wave

$$
v_{ \pm}(x, k)=-\int_{E_{3}} \frac{\exp \{\mp i|k| \cdot|x-y|\}}{4 \pi|x-y|} b_{2}(y) \psi_{ \pm}(y, k) d y
$$

does not always satisfy the Sommerfeld radiation condition (2.10)

12) Cf. Ikebe [5]; Theorem 3. 
for $\kappa=\mp|k|$. The radiation condition is satisfied if we assume the stronger condition $\left(A_{1}\right)$ :

$$
(1+|x|)^{(3+\delta) / 2} q(x) \in L^{2}\left(E_{3}\right), \quad \delta>0 .
$$

\section{§5. Expansion theorem.}

Let us consider the bi-linear form $E_{e}(f, g)$ in $\mathfrak{S}$ given by

$$
E_{e}(f, g)=\lim _{\varepsilon \rightarrow+0} \frac{1}{2 \pi i} \int_{e}(\{R(\lambda+i \varepsilon)-R(\lambda-i \varepsilon)\} f, g) d \lambda \quad(f, g \in \mathfrak{E})
$$

for a subinterval $e=(\alpha, \beta)$ of $(-\infty, \infty)$. We denote by $\widetilde{\boldsymbol{\Gamma}}_{+}$the class of (possibly infinite) subintervals $e=(\alpha, \beta)$ of $(-\infty, \infty)$ such that the closure of $\{\sqrt{\lambda+i 0} ; \lambda \in e\}$ does not contain any point of $\Sigma$, and by $\widetilde{\Gamma}_{-}$the class of $e$ such that the closure of $\{\sqrt{\lambda-i 0} ; \lambda \in e\}$ does not contain any point of $\Sigma$, where $\sqrt{\lambda \pm i 0}= \pm \sqrt{\bar{\lambda}}$ if $\lambda>0$, and $=i \sqrt{-\lambda}$ if $\lambda<0$. Note that if we choose $\alpha$ sufficiently large, then both $(\alpha, \infty)$ and $(-\infty,-\alpha)$ belong with any of their subintervals to $\widetilde{\Gamma}_{+} \cap \widetilde{\Gamma}_{-}$since $\Sigma$ forms a bounded set in $\operatorname{Im} \kappa \geq 0$ (see (1) of Theorem 2.1). Our first aim in this $\S$ is to show that (5.1) defines for each $e \in \widetilde{\Gamma}_{+} \cap \widetilde{\Gamma}_{-}$a bounded bi-linear form in $\mathfrak{S}$.

We begin with proving the following lemma due essentially to Kato [13].

Lemma 5.1. Let $a(x)=a_{1}(x)=|q(x)|^{1 / 2}$. Then we have the following inequalities : for $f \in \mathfrak{S}$

$$
\int_{-\infty}^{\infty}\left\{\left\|A R_{0}(\lambda+i \varepsilon) f\right\|^{2}+\left\|A R_{0}(\lambda-i \varepsilon) f\right\|^{2}\right\} d \lambda \leq C_{A}\|f\|^{2},
$$

$(5.3)_{+} \quad \int_{e}\|A R(\lambda+i \varepsilon) f\|^{2} d \lambda \leq C_{A, e}\|f\|^{2}, \quad$ if $\quad e=(\alpha, \beta) \in \widetilde{\Gamma}_{+}$,

$$
\int_{e}\left\|\left.A R(\lambda-i \varepsilon) f\right|_{i} ^{2} d \lambda \leq C_{A, e}\right\| f \|^{2}, \quad \text { if } \quad e=(\alpha, \beta) \in \widetilde{\Gamma}_{-},
$$

where $C_{A}$ and $C_{A, e}$ are positive constants independent of $\varepsilon>0$ sufficiently small. Moreover, there exist the strong limits as $\varepsilon \rightarrow+0$ of $A R_{0}(\lambda \pm i \varepsilon) f$ and $A R(\lambda \pm i \varepsilon) f$ respectively in $L^{2}(-\infty, \infty ; \mathfrak{S})$ and $L^{2}(e ; \mathfrak{S}), e \in \widetilde{\Gamma}_{ \pm}$, for any $f \in \mathfrak{S}$. If we denote the limits by $A R_{0}(\lambda \pm i 0) f$ and $A R(\lambda \pm i 0) f$, then they also satisfy (5.2) and (5.3) $)_{ \pm}$with the same constants. 
Proof. The assertions for $A R_{0}(\lambda \pm i \varepsilon) f$ are already proved by Kato under a more general assumption on $q(x)$ (it is sufficient to assume $q(x) \in L^{3 / 2}\left(E_{3}\right)$, see [13]; Theorems 5.1, 6.1, and Remark 6.5). For $A R(\lambda \pm i \varepsilon) f$, we have in virtue of (2.11)

$$
\text { (5.4) } A R(\lambda \pm i \varepsilon)=\left\{I+Q(\sqrt{\lambda \pm i \varepsilon)}\}^{-1} A R_{0}(\lambda \pm i \varepsilon), \quad \operatorname{Im} \sqrt{\lambda \pm i \varepsilon}>0 .\right.
$$

For a fixed $e=(\alpha, \beta) \in \widetilde{\Gamma}_{ \pm}$, there exists a small $\varepsilon_{0}>0$ such that the closure of $N_{e}^{ \pm}=\left\{\kappa=\sqrt{\lambda \pm i \varepsilon} ; \lambda \in(\alpha, \beta), \varepsilon \in\left(0, \varepsilon_{0}\right)\right\}$ does not intersect with $\Sigma$. So, by (3) of Theorem 2.1, $\left\{I+Q_{0}(\sqrt{\lambda \pm i \varepsilon})\right\}^{-1}$ is bounded, continuous in $(\lambda, \varepsilon) \in(\alpha, \beta) \times\left(0, \varepsilon_{0}\right)$, and converges as $\varepsilon \rightarrow+0$ to $\left\{I+Q_{0}(\sqrt{\lambda \pm i 0})\right\}^{-1}$ in the sense of the uniform topology of operators, where $\sqrt{\lambda \pm i 0}= \pm \sqrt{\lambda}$ if $\lambda \geq 0$, and $=i \sqrt{-\lambda}$ if $\lambda<0$. Thus $A R(\lambda \pm i \varepsilon) f$ satisfies (5.3) $)_{ \pm}$with $C_{A, e}=C_{A} \cdot \sup _{\lambda \in(\alpha, \beta), \mathbb{\varepsilon} \in\left(0, \varepsilon_{0}\right)}\left\|\left\{I+Q_{0}(\sqrt{\lambda \pm i \varepsilon})\right\}^{-1}\right\|^{2}$, and converges as $\varepsilon \rightarrow+0$ to $A R(\lambda \pm i 0) f$ in $L^{2}(e ; \mathfrak{E})$. $\quad$ Q. E. D.

Using this lemma, we can prove the

Lemma 5. 2. ${ }^{13)}$ For each $e=(\alpha, \beta)$ in $\widetilde{\Gamma}_{+} \cap \widetilde{\Gamma}_{-}$(5.1) defines $a$ bounded bilinear form in $\mathfrak{S}$. Hence, by the Riez theorem, there exists a bounded operator E(e) satisfying

$$
(E(e) f, g)=\lim _{\varepsilon \rightarrow+0} \frac{1}{2 \pi i} \int_{e}(\{R(\lambda+i \varepsilon)-R(\lambda-i \varepsilon)\} f, g) d \lambda \quad(f, g \in \mathfrak{E}) .
$$

In particular

$$
E(e)=0 \quad \text { if } \quad e=(\alpha, \beta) \subset(-\infty, 0) .
$$

Proof. It follows from the resolvent equation (1.6) that

$$
\begin{aligned}
& R(\lambda+i \varepsilon)-R(\lambda-i \varepsilon) \\
= & R_{0}(\lambda+i \varepsilon)-R_{0}(\lambda-i \varepsilon)-R_{0}(\lambda+i \varepsilon) V R(\lambda+i \varepsilon)+R_{0}(\lambda-i \varepsilon) V R(\lambda-i \varepsilon) .
\end{aligned}
$$

Substituting this in (5.1) and noting the relation

$$
\lim _{\varepsilon \rightarrow+0} \frac{1}{2 \pi i} \int_{e}\left(\left\{R_{0}(\lambda+i \varepsilon)-R_{0}(\lambda-i \varepsilon)\right\} f, g\right) d \lambda=\left(E_{0}(e) f, g\right),
$$

where $E_{0}(e)=E_{0, \beta}-E_{0, \alpha}$, we have for $f, g$ in $\mathfrak{S}$

13) Cf. J. Schwartz [10]. He obtained results in which $q(x) \in L^{1} \cap L^{\infty}$ was assumed together with the existence of an $e \in \tilde{\Gamma}_{+} \cap \tilde{\Gamma}_{-}$. 
(5. 8) $\quad E_{e}(f, g)=\left(E_{0}(e) f, g\right)-\lim _{\varepsilon \rightarrow 0} \frac{1}{2 \pi i} \int_{e}\left(A R(\lambda+i \varepsilon) f, B^{*} R_{0}(\lambda+i \varepsilon)^{*} g\right) d \lambda$

$$
+\lim _{\varepsilon \rightarrow+0} \frac{1}{2 \pi i} \int_{e}\left(A R(\lambda-i \varepsilon) f, B^{*} R_{0}(\lambda-i \varepsilon)^{*} g\right) d \lambda .
$$

We have factored $V$ as $B A$ choosing $a(x)=a_{1}(x)$. Then, since $\left\|B^{*} R_{0}(\lambda \pm i \varepsilon)^{*} g\right\|=\left\|A R_{0}(\lambda \mp i \varepsilon) g\right\|$, we can use the above lemma to see that the limits in the right member of (5.8) exist and are estimated as

$$
\left|\lim _{\varepsilon \rightarrow+0} \frac{1}{2 \pi i} \int_{e}\left(A R(\lambda \pm i \varepsilon) f, B^{*} R_{0}(\lambda \pm i \varepsilon)^{*} g\right) d \lambda\right| \leq \text { const }\|f\| \cdot\|g\| \text {. }
$$

Hence we conclude that $E_{e}(f, g)$ is a bounded bi-linear form in $\mathfrak{S}_{\text {. }}$. (5.6) is evident from (5.8) if we note that both $R_{0}(\zeta)$ and $R(\zeta)$ are analytic in a neighborhood of $e \in \widetilde{\Gamma}_{+} \cap \widetilde{\Gamma}_{-}$when $e \subset(-\infty, 0)$. Q.E.D.

We put $\Gamma=\Gamma_{+} \cap \Gamma_{-}$, where

$$
\Gamma_{+}=\left\{e \in \widetilde{\Gamma}_{+} ; e \subset(0, \infty)\right\}, \quad \Gamma_{-}=\left\{e \in \widetilde{\Gamma}_{-} ; e \subset(0, \infty)\right\}
$$

Then $e \in \Gamma$ if and only if its closure does not contain any point of $\sigma_{s}(L)$. We shall show that $E(e), e \in \Gamma$ constitutes the so-called "spectral projection" of $L$, and that an arbitrary function in $E(e) \mathfrak{S}$ is expanded in terms of the distorted plane waves and the adjoint waves.

The following lemma can be proved by the same reasoning as in the proof of the above lemma.

Lemma 5.3. There exist bounded operators $X_{\mp}(e)\left(e \in \Gamma_{\mp}\right)$ and $Y_{ \pm}\left(e^{\prime}\right)\left(e^{\prime} \in \Gamma_{ \pm}\right)$satisfying the following relations for $f, g$ in $\mathfrak{S}$.

$$
\begin{aligned}
& \left(X_{ \pm}(e) f, g\right) \\
= & \lim _{\varepsilon \rightarrow+0} \frac{1}{2 \pi i} \int_{e}\left(\left\{R_{0}(\lambda+i \varepsilon)-R_{0}(\lambda-i \varepsilon)\right\} f, V^{*} R(\lambda \mp i \varepsilon)^{*} g\right) d \lambda,
\end{aligned}
$$

$$
\begin{aligned}
& \left(Y_{ \pm}\left(e^{\prime}\right) f, g\right) \\
= & \lim _{\varepsilon \rightarrow+0} \frac{1}{2 \pi i} \int_{e^{\prime}}\left(\left\{R_{0}(\lambda+i \varepsilon)-R_{0}(\lambda-i \varepsilon)\right\} V R(\lambda \pm i \varepsilon) f, g\right) d \lambda .
\end{aligned}
$$

We define the bounded operators $W_{ \pm}(e)\left(e \in \Gamma_{\mp}\right)$ and $Z_{ \pm}\left(e^{\prime}\right)\left(e^{\prime} \in \Gamma_{ \pm}\right)$ by

$$
W_{ \pm}(e)=E_{0}(e)-X_{ \pm}(e), \quad Z_{ \pm}\left(e^{\prime}\right)=E_{0}\left(e^{\prime}\right)-Y_{ \pm}\left(e^{\prime}\right),
$$

and define the transforms $\hat{f}_{ \pm}$and $\hat{f}_{ \pm}^{*}$ of $f$ in $C_{0}^{\infty}\left(E_{3}\right)$ by 


$$
\begin{array}{ll}
\hat{f}_{ \pm}(k)=(2 \pi)^{-3 / 2} \int_{E_{3}} \overline{\varphi_{ \pm}(x, k)} f(x) d x, & \mp \mid k i \notin \Sigma, \\
\hat{f}_{ \pm}^{*}(k)=(2 \pi)^{-3 / 2} \int_{E_{3}} \overline{\varphi_{ \pm}^{*}(x, k)} f(x) d x, & \pm|k| \notin \Sigma,
\end{array}
$$

where $\varphi_{ \pm}(x, k)$ and $\varphi_{ \pm}^{*}(x, k)$ are defined by (4.13) and (4.14), respectively.

Lemma 5.4. (1) Let $K_{e}$ denote the domain in $E_{3}$ such that $K_{e}=\left\{k \in E_{3} ;|k|^{2} \in e\right\}$. Then we have

$$
\begin{array}{ll}
\int_{K_{e}}\left|\hat{f}_{ \pm}(k)\right|^{2} d k \leq C_{e}\|f\|^{2} \quad & \left(e \in \Gamma_{\mp}\right), \\
\int_{K_{e^{\prime}}}\left|\hat{f}_{ \pm}^{*}(k)\right|^{2} d k \leq C_{e^{\prime}}\|f\|^{2} \quad\left(e^{\prime} \in \Gamma_{ \pm}\right) .
\end{array}
$$

Hence, we can extend the maps $f \rightarrow \hat{f}_{ \pm}$and $f \rightarrow \hat{f}_{ \pm}^{*}$ to continuous maps of $\mathfrak{S}$ into $L^{2}\left(K_{e}\right)$ and $L^{2}\left(K_{e^{\prime}}\right)$, ${ }^{14)}$ respectively, taking the integrals (5.13) and (5.14) in the sense of the limit in the mean.

(2) $W_{ \pm}(e)$ and $Z_{ \pm}\left(e^{\prime}\right)$ are represented as

(5. 16) $\left[W_{ \pm}(e) f\right](x)=(2 \pi)^{-3 / 2} \int_{K_{e}} \varphi_{ \pm}(x, k) \hat{f}(k) d k$,

(5. 17) $\left[Z_{ \pm}\left(e^{\prime}\right) f\right](x)=(2 \pi)^{-3 / 2} \int_{K_{e^{\prime}}} \exp \{i k \cdot x\} \hat{f}_{ \pm}^{*}(k) d k$,

for a.e. $x \in E_{3}$, where $\hat{f}$ denotes the Fourier transform of $f \in \mathfrak{S}$ :

$$
\hat{f}(k)=(2 \pi)^{--3 / 2} \int_{E_{3}} f(x) \exp \{-i k \cdot x\} d x .
$$

Proof. Since $W_{ \pm}(e)$ and $Z_{ \pm}\left(e^{\prime}\right)$ are both bounded operators, and $C_{0}^{\infty}\left(E_{3}\right)$ is dense in $\mathfrak{S},(5.15),(5.16)$ and $(5.17)$ are immediate if we show the following relations for $f, g$ in $C_{0}^{\infty}\left(E_{3}\right)$ :

(5. 19) $\quad\left(W_{ \pm}(e) f, g\right)=\int_{K_{\mathrm{f}}} \hat{f}(k) \overline{\hat{g}_{ \pm}(k)} d k, \quad\left(Z_{ \pm}\left(e^{\prime}\right) f, g\right)=\int_{K_{\ell^{\prime}}} \hat{f}_{ \pm}^{*}(k) \overline{\hat{g}(k)} d k$

Moreover, we have only to show (5.19) in the case when $e$ and $e^{\prime}$ are finite. For, if $e=(\alpha, \infty), e^{\prime}=\left(\alpha^{\prime}, \infty\right)$, then we can choose $e_{n}=\left(\alpha, \beta_{n}\right)$, $e_{n}^{\prime}=\left(\alpha^{\prime}, \beta_{n}\right)$ such that $\beta_{n} \rightarrow \infty$ and $W_{ \pm}\left(e_{n}\right) \rightarrow W_{ \pm}(e), Z_{ \pm}\left(e_{n}^{\prime}\right) \rightarrow Z_{ \pm}\left(e^{\prime}\right)$ in

14) We shall prove later in Theorem 5.1 that these are onto maps. 
the weak topology of operators.

First note that for each $e \subset(0, \infty) E_{0}(e)$ can be represented as

$$
\left[E_{0}(e) f\right](x)=(2 \pi)^{-3 / 2} \int_{K_{e}} \exp \{i k \cdot x\} \hat{f}(k) d k
$$

(Fourier inversion formula).

Applying the Parseval equality to (5.10), we have for $e \in \Gamma_{\mp}$

$$
\begin{aligned}
& \left(X_{ \pm}(e) f, g\right) \\
= & \lim _{\varepsilon \rightarrow+0} \frac{1}{2 \pi i} \int_{e} d \lambda \int_{E_{3}} \frac{2 i \varepsilon}{\left(\lambda-|k|^{2}\right)^{2}+\varepsilon^{2}} \hat{f}(k)\left[\overline{\left.V^{*} R(\lambda \mp i \varepsilon)^{*} g\right]^{\wedge}(k)} d k .\right.
\end{aligned}
$$

Here we choose $f, g$ from $C_{0}^{\infty}\left(E_{3}\right)$. Then, by the Fubini theorem, it follows that

$$
\begin{aligned}
& {\left[V^{*} R(\lambda \mp i \varepsilon)^{*} g\right]^{\wedge}(k) } \\
= & (2 \pi)^{-3 / 2} \int_{E_{3}} \exp \{-i k \cdot x\} \overline{q(x)} d x \int_{E_{3}} \overline{R(y, x ; \sqrt{\lambda \mp i \varepsilon})} g(y) d y \\
= & (2 \pi)^{-3 / 2} \int_{E_{3}} g(y) d y \overline{\int_{E_{3}} R(y, x ; \sqrt{\lambda \mp i \varepsilon}) b_{2}(x) a_{2}(x) \exp \{i k \cdot x\} d x .} .
\end{aligned}
$$

Noting the relation

$$
\begin{aligned}
R(\zeta) B= & R_{0}(\zeta) B\{I-A R(\zeta) B\} \\
& \subset R_{0}(\zeta) B-R_{0}(\zeta) B\left\{I+Q_{0}(\sqrt{\zeta}\}^{-1} A R_{0}(\zeta) B\right. \\
= & R_{0}(\zeta) B-R_{0}(\zeta) B\left\{I+Q_{0}(\sqrt{\zeta})\right\}^{-1}\left\{I+A R_{0}(\zeta) B\right\} \\
& +R_{0}(\zeta) B\left\{I+Q_{0}(\sqrt{\zeta})\right\}^{-1} \\
= & R_{0}(\zeta) B\left\{I+Q_{0}(\sqrt{\zeta})\right\}^{-1},
\end{aligned}
$$

we have from (4.5), (4.6) and (4.7)

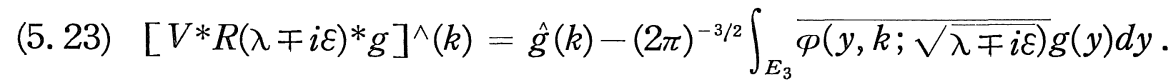

Substitute this in (5.21). Then we can once again use the Fubini theorem and obtain for $f, g$ in $C_{0}^{\infty}\left(E_{3}\right)$

$$
\begin{aligned}
&\left(X_{ \pm}(e) f, g\right) \\
&= \lim _{\varepsilon \rightarrow+0} \int_{E_{2}} \hat{f}(k) d k \frac{1}{2 \pi i} \int_{e} \frac{2 i \varepsilon}{\left(\lambda-|k|^{2}\right)^{2}+\varepsilon^{2}}\{\overline{\hat{g}(k)}-\overline{\Psi(k ; \sqrt{\lambda \mp i \varepsilon})}\} d \lambda ; \\
& \Psi(k ; \sqrt{\lambda \mp i \varepsilon})=(2 \pi)^{-3 / 2} \int_{E_{3}} \overline{\rho(y, k ; \sqrt{\lambda \mp i \varepsilon)} g(y) d y .}
\end{aligned}
$$


By Lemma 4.2 we see that $\Psi(k ; \sqrt{\lambda \mp i \varepsilon})$ is bounded, continuous in $k \in E_{3}, \lambda \in e$, and $\varepsilon>0$ sufficiently small, and tends as $\sqrt{\lambda \mp i \varepsilon} \rightarrow \mp|k|$ to $\hat{g}_{ \pm}(k)$. Hence the bounded convergence theorem shows that

$$
\left(X_{ \pm}(e) f, g\right)=\int_{K_{e}} \hat{f}(k) \overline{\hat{g}(k)} d k-\int_{K_{e}} \hat{f}(k) \overline{\hat{g}_{ \pm}(k)} d k,
$$

where we have used the well known relation

$$
\lim _{\varepsilon \rightarrow+0} \frac{1}{2 \pi i} \int_{e} \frac{2 i \varepsilon}{\left(\lambda-|k|^{2}\right)^{2}+\varepsilon^{2}} f(\lambda, \varepsilon) d \lambda=\left\{\begin{array}{lll}
0 & \text { if } & |k|^{2} \notin e \\
f\left(|k|^{2}, 0\right) & \text { if } & |k|^{2} \in e,
\end{array}\right.
$$

in which $f(\lambda, \varepsilon)$ is a continuous functions of $\lambda \in e$ and $\varepsilon \in\left[0, \varepsilon_{0}\right]$ $\left(\varepsilon_{0}>0\right)$.

By virtue of (5.20) and (5.24), we get the first relation of (5.19) for each finite $e \in \Gamma_{\mp}$ and $f, g \in C_{0}^{\infty}\left(E_{3}\right)$. The second relation can be proved quite in the same way.

Q.E.D.

We can now prove the

Lemma 5.5. If $e$ is in $\Gamma=\Gamma_{+} \cap \Gamma_{-}$, then both $W_{ \pm}(e)$ and $Z_{ \pm}(e)$ can be defined by (5.12), and we have

$$
\begin{aligned}
& E(e)=W_{ \pm}(e) Z_{ \pm}(e), \\
& E_{0}(e)=Z_{ \pm}(e) W_{ \pm}(e) .
\end{aligned}
$$

Proof. The resolvent equation

$$
R(\lambda+i \varepsilon)-R(\lambda-i \varepsilon)=2 i \varepsilon R(\lambda \mp i \varepsilon) R(\lambda \pm i \varepsilon)=2 i \varepsilon R(\lambda \mp i \varepsilon) R^{*}(\lambda \mp i \varepsilon)^{*}
$$

implies that

$$
\begin{aligned}
& R(x, y ; \sqrt{\lambda+i \varepsilon})-R(x, y ; \sqrt{\lambda-i \varepsilon}) \\
= & 2 i \varepsilon \int_{E_{3}} R\left(x, z ; \sqrt{\lambda \mp i \varepsilon)} \overline{R^{*}(y, z ; \sqrt{\bar{\lambda} \mp i \varepsilon)}} d z .\right.
\end{aligned}
$$

Making use of the Parseval equality, and taking (4.10) and (4.11) into account, we obtain

$$
\begin{aligned}
& R(x, y ; \sqrt{\overline{\lambda+i \varepsilon})}-R(x, y ; \sqrt{\overline{\lambda-i \varepsilon}}) \\
= & (2 \pi)^{-3} \int_{E_{3}} \frac{2 i \varepsilon \varphi\left(x, k ; \sqrt{\lambda \mp i \varepsilon)} \overline{\varphi^{*}(y, k ; \sqrt{\lambda \mp i \varepsilon})} d k .\right.}{\left(\lambda-|k|^{2}\right)^{2}+\varepsilon^{2}} .
\end{aligned}
$$

Substitute this in (5.5). Then we have for $f, g$ in $C_{0}^{\infty}\left(E_{3}\right)$ 


$$
\begin{aligned}
& (E(e) f, g)=\lim _{\varepsilon \rightarrow+0} \frac{1}{2 \pi i} \int_{e} d \lambda \int_{E_{3}} \frac{2 i \varepsilon}{\left(\lambda-|k|^{2}\right)^{2}+\varepsilon^{2}} \Psi(k ; \sqrt{\lambda \mp i \varepsilon}) \\
& \times \overline{\Psi^{*}(k ; \sqrt{\lambda \pm i \varepsilon})} d k \\
& \Psi^{*}(k ; \sqrt{\lambda \mp i \varepsilon})=(2 \pi)^{-3 / 2} \int_{E_{3}} \overline{\phi^{*}(x, k ; \sqrt{\lambda \mp i \varepsilon})} f(x) d x,
\end{aligned}
$$

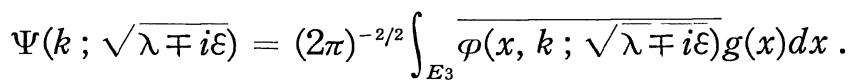

By the same reasoning as in the proof of Lemma 5.4, we find for each finite $e$ in $\Gamma$ the following relation:

$$
(E(e) f, g)=\int_{K_{e}} \hat{f}_{ \pm}^{*}(k) \overline{\hat{g}_{ \pm}(k)} d k, f, g \in C_{0}^{\infty}\left(E_{3}\right) .
$$

It is already proved in Lemma 5.4 that both $\hat{f}_{ \pm}^{*}$ and $\hat{g}_{ \pm}$are in $L^{2}\left(K_{e}\right)$ for any $e$ in $\Gamma$. So, we can extend (5.27) to infinite $e$. Since $C_{0}^{\infty}\left(E_{3}\right)$ is dense in $\mathfrak{S},(5.27)$ proves with expression (5.16) and (5.17) the first relation (5.25).

Next we proceed to derive (5.26). It follows from (5.12) that

$$
Z_{ \pm}(e) W_{ \pm}(e)=E_{* 0}(e)-Y_{ \pm}(e) E_{0}(e)-E_{0}(e) X_{ \pm}(e)+Y_{ \pm}(e) X_{ \pm}(e) \text {. }
$$

So, we have only to show the relation

$$
Y_{ \pm}(e) X_{ \pm}(e)=Y_{ \pm}(e) E_{0}(e)+E_{0}(e) X_{ \pm}(e) .
$$

For the sake of simplicity, we put

$$
\begin{aligned}
& v_{\varepsilon}(x, k)=\varphi\left(x, k ; \sqrt{|k|^{2}+i \varepsilon}\right)-\exp \{i k \cdot x\}, \\
& v_{\varepsilon}^{*}(x, k)=\phi^{*}\left(x, k ; \sqrt{|k|^{2}+i \varepsilon}\right)-\exp \{i k \cdot x\}, \\
& h_{\varepsilon}(x, k)=q(x) \varphi\left(x, k ; \sqrt{|k|^{2}+i \varepsilon}\right), \\
& h_{\varepsilon}^{*}(x, k)=\overline{q(x)} \varphi^{*}\left(x, k ; \sqrt{\left.|k|^{2}+i \varepsilon\right)} .\right.
\end{aligned}
$$

Then these functions are all in $L^{2}\left(E_{3}\right)$ as functions of $x$ for each $|k|^{2} \in e$ and $|\varepsilon| \neq 0$ sufficiently small. It follows from (4.9) that

$$
v_{\varepsilon}=-R_{0}\left(|k|^{2}+i \varepsilon\right) h_{\varepsilon}, \quad v_{\varepsilon}^{*}=-R_{0}\left(|k|^{2}+i \varepsilon\right) h_{\varepsilon}^{*} .
$$

We put

$$
\begin{aligned}
& \tau_{\varepsilon}(x)=(2 \pi)^{-3 / 2} \int_{K_{e}} v_{\varepsilon}(x, k) \hat{f}(k) d k, \\
& \tau_{\varepsilon}^{*}(x)=(2 \pi)^{-3 / 2} \int_{K_{e}} v_{\varepsilon}^{*}(x, k) \hat{g}(k) d k,
\end{aligned}
$$


for $\hat{f}, \hat{g}$ in $C_{0}^{\infty}\left(K_{e}\right)$. Then, as $\varepsilon \rightarrow \mp 0$, we have, taking Lemma 4.2 and (5.24) into account,

(5. 30) $\tau_{\varepsilon}(x) \rightarrow-\left[X_{ \pm}(e) f\right](x), \quad \tau_{\varepsilon}^{*}(x) \rightarrow-\left[Y_{ \pm}(e)^{*} g\right](x), \quad$ a.e. $x \in E_{3}$.

By the Fubini theorem

$$
\begin{aligned}
& \int_{E_{3}} \tau_{\varepsilon}(x) \overline{\tau_{\varepsilon^{\prime}}^{*}(x)} d x \\
& \quad=(2 \pi)^{-3} \int_{K_{e}} \hat{f}(k) d k \int_{K_{e}} \overline{\hat{g}\left(k^{\prime}\right)} d k^{\prime} \int_{E_{3}} v_{\varepsilon}(x, k) \overline{v_{\varepsilon}^{* \prime}\left(x, k^{\prime}\right)} d x \\
& \quad=(2 \pi)^{-3} \int_{K_{e}} \hat{f}(k) d k \int_{K_{e}} \overline{\hat{g}\left(k^{\prime}\right)} d k^{\prime}\left(R_{0}\left(|k|^{2}+i \varepsilon\right) h_{\varepsilon}, R_{0}\left(\left|k^{\prime}\right|^{2}+i \varepsilon^{\prime}\right) h_{\varepsilon}^{* \prime}\right) \equiv(*) .
\end{aligned}
$$

Using the resolvent equation $R_{0}\left(\overline{\zeta^{\prime}}\right) R_{0}(\zeta)=\left(\overline{\zeta^{\prime}}-\zeta\right)^{-1}\left\{R_{0}\left(\overline{\zeta^{\prime}}\right)-R_{0}(\zeta)\right\}$, we have

$$
(*)=(2 \pi)^{-3} \int_{K_{e}} \hat{f}(k) d k \int_{K_{e}} \overline{\hat{g}\left(k^{\prime}\right)} d k^{\prime} \frac{\left(h_{\varepsilon}, v_{\varepsilon}^{* \prime}\right)-\left(v_{\varepsilon}, h_{\varepsilon}^{* \prime}\right)}{\left|k^{\prime}\right|^{2}-|k|^{2}-i\left(\varepsilon^{\prime}+\varepsilon\right)}
$$

by (5.29). Here we can use the Fubini theorem to exchange the order of the integration. Then

$$
\begin{aligned}
(*)= & (2 \pi)^{-3} \int_{E_{3}} d x\left\{\int_{K_{e}} h_{\varepsilon}(x, k) \hat{f}(k) d k \overline{\int_{K_{e}} \frac{v_{\varepsilon}^{* \prime}\left(x, k^{\prime}\right) \hat{g}\left(k^{\prime}\right)}{\left|k^{\prime}\right|^{2}-|k|^{2}+i\left(\varepsilon^{\prime}+\varepsilon\right)} d k^{\prime}}\right. \\
& \left.-\int_{K_{e}} \frac{v_{\varepsilon}(x, k) \hat{f}(k)}{\left|k^{\prime}\right|^{2}-|k|^{2}-i\left(\varepsilon^{\prime}+\varepsilon\right)} d k \int_{K_{e}} \overline{h_{\varepsilon}^{* \prime}\left(x, k^{\prime}\right) \hat{g}\left(k^{\prime}\right)} d k^{\prime}\right\},
\end{aligned}
$$

where we have, taking (4.2) into account,

$$
\begin{gathered}
\int_{K_{e}} \frac{v_{\varepsilon}^{* \prime}\left(x, k^{\prime}\right) \hat{g}\left(k^{\prime}\right)}{\left|k^{\prime}\right|^{2}-|k|^{2}+i\left(\varepsilon^{\prime}+\varepsilon\right)} d k^{\prime}=\int_{K_{e}} \frac{\varphi^{*}\left(x, k^{\prime} ; \sqrt{\left|k^{\prime}\right|^{2}+i \varepsilon^{\prime}}\right) \hat{g}\left(k^{\prime}\right)}{\left|k^{\prime}\right|^{2}-|k|^{2}+i\left(\varepsilon^{\prime}+\varepsilon\right)} d k^{\prime} \\
-(2 \pi)^{3 / 2}\left[R_{0}\left(|k|^{2}-i\left(\varepsilon^{\prime}+\varepsilon\right)\right) g\right](x), \\
\int_{K_{e}} \frac{v_{\varepsilon}(x, k) \hat{f}(k)}{\left|k^{\prime}\right|^{2}-|k|^{2}-i\left(\varepsilon^{\prime}+\varepsilon\right)} d k=\int_{K_{e}} \frac{\varphi\left(x, k ; \sqrt{\left.|k|^{2}+i \varepsilon\right)} \hat{f}(k)\right.}{\left|k^{\prime}\right|^{2}-|k|^{2}-i\left(\varepsilon^{\prime}+\varepsilon\right)} d k^{\prime} \\
+(2 \pi)^{3 / 2}\left[R_{0}\left(\left|k^{\prime}\right|^{2}-i\left(\varepsilon^{\prime}+\varepsilon\right)\right) f\right](x),
\end{gathered}
$$

since $\hat{f}, \hat{g}$ are in $C_{0}^{\infty}\left(K_{e}\right)$. Noting (5.29) and exchanging the integration order, we finally get

$$
\begin{aligned}
& \int_{E_{3}} \tau_{\varepsilon}(x) \overline{\tau_{\varepsilon}^{*}(x)} d x \\
& \quad=-(2 \pi)^{-3 / 2} \int_{K_{e}} \hat{f}(k) d k \int_{E_{3}} h_{\varepsilon}(x, k) \overline{\left[R_{0}\left(|k|^{2}-i\left(\varepsilon^{\prime}+\varepsilon\right)\right) g\right](x)} d x
\end{aligned}
$$




$$
\begin{aligned}
& -(2 \pi)^{-3 / 2} \int_{K_{e}} \overline{\hat{g}\left(k^{\prime}\right)} d k^{\prime} \int_{E_{3}}\left[R_{0}\left(\left|k^{\prime}\right|^{2}-i\left(\varepsilon^{\prime}+\varepsilon\right)\right) f\right](x) \overline{h_{\varepsilon}^{* \prime}\left(x, k^{\prime}\right)} d x \\
= & -(2 \pi)^{-3 / 2} \int_{E_{3}}\left\{\int_{K_{e}}\left[R_{0}\left(|k|^{2}+i\left(\varepsilon^{\prime}+\varepsilon\right)\right) h_{\varepsilon}\right](x, k) \hat{f}(k) d k\right\} \overline{g(x)} d x \\
& -(2 \pi)^{-3 / 2} \int_{E_{3}} f(x)\left\{\int_{K_{e}}\left[R_{0}\left(\left|k^{\prime}\right|^{2}+i\left(\varepsilon^{\prime}+\varepsilon\right)\right) h_{\varepsilon}^{*}\right]\left(x, k^{\prime}\right) \hat{g}\left(k^{\prime}\right) d k^{\prime}\right\} d x .
\end{aligned}
$$

We make $\varepsilon \rightarrow \mp 0$ and $\varepsilon^{\prime} \rightarrow \pm 0$. Then

$$
\begin{aligned}
& {\left[R_{0}\left(|k|^{2}+i\left(\varepsilon^{\prime}+\varepsilon\right)\right) h_{\varepsilon}\right](x, k) \rightarrow v_{ \pm}(x, k)=\varphi_{ \pm}(x, k)-\exp \{i k \cdot x\},} \\
& {\left[R_{0}\left(\left|k^{\prime}\right|^{2}+i\left(\varepsilon^{\prime}+\varepsilon\right)\right) h_{\varepsilon^{\prime}}^{*}\right]\left(x, k^{\prime}\right) \rightarrow v_{ \pm}^{*}\left(x, k^{\prime}\right)=\varphi_{ \pm}^{*}\left(x, k^{\prime}\right)-\exp \left\{i k^{\prime} \cdot x\right\} .}
\end{aligned}
$$

Thus we obtain, using the Lebesgue theorem,

$$
\begin{gathered}
\int_{E_{3}}\left[X_{ \pm}(e) f\right](x) \overline{\left[Y_{ \pm}(e)^{*} g\right](x)} d x=\int_{E_{3}}\left[X_{ \pm}(e) f\right](x) \overline{g(x)} d x \\
+\int_{E_{3}} f(x) \overline{\left[Y_{ \pm}(e)^{*} g\right](x)} d x
\end{gathered}
$$

in virtue of (5.24) and (5.30). This implies (5.28) if we note that $\left\{f ; \hat{f} \in C_{0}^{\infty}\left(K_{e}\right)\right\}$ is dense in $E_{0}(e) \mathfrak{S}=\left\{f ; \hat{f} \in L^{2}\left(K_{e}\right)\right\}$ Q. E. D.

It remains for us to add a few more facts in order to prove the following expansion theorem.

Theorem 5.1.15) (1) (spectral projection) For each $e$ in $\Gamma$, the bounded operator $E(e)$ defined in Lemma 5.2 gives the "spectral projection" (not necessary orthogonal) of L:

$$
E(e)^{2}=E(e), \quad E(e) L \subset L E(e) .
$$

(2) (similarity between $L_{0}$ and $L$ ) The following relations hold for each $e$ in $\Gamma$, and $\zeta$ in the resolvent set of $L$.

$$
R(\zeta) W_{ \pm}(e)=W_{ \pm}(e) R_{0}(\zeta), \quad R_{0}(\zeta) Z_{ \pm}(e)=Z_{ \pm}(e) R(\zeta) .
$$

Hence the parts of $L_{0}$ and $L$ respectively in $E_{0}(e) \mathfrak{E}$ and $E(e) \mathfrak{S}$ are similar to each other:

$$
L E(e) \subset W_{ \pm}(e) L_{0} E_{0}(e) W_{ \pm}(e)^{-1}, \quad W_{ \pm}(e)^{-1}=Z_{ \pm}(e) .
$$

Moreover, we have

$$
\mathscr{D}(L) \cap E(e) \mathfrak{W}=E(e) \mathscr{D}(L)
$$

15) Cf. Ikebe [5]; Theorem 5 and Povzner [3]; Theorem 1. 
(3) (generalized Fourier inversion formula) The transform

$$
\hat{f}_{ \pm}^{*}(k)=(2 \pi)^{-3 / 2} \int_{E_{3}} \overline{\varphi_{ \pm}^{*}(x, k)} f(x) d x
$$

gives a bounded map of $E(e) \mathfrak{S}$ one-to-one onto $L^{2}\left(K_{e}\right)$ for each $e$ in $\Gamma$. We have the following inversion formula:

$$
[E(e) f](x)=(2 \pi)^{-3 / 2} \int_{K_{e}} \varphi_{ \pm}(x, k) \hat{f}_{ \pm}^{*}(k) d k .
$$

(4) (diagonal representation of $L) f(x) \in E(e) \mathscr{D}(L)$ if and only if $|k|^{2} \hat{f}_{ \pm}^{*}(k) \in L^{2}\left(K_{e}\right)$. We have the following representation of $L$ :

$$
[L E(e) f](x)=(2 \pi)^{-3 / 2} \int_{K_{e}}|k|^{2} \phi_{ \pm}(x, k) \hat{f}_{ \pm}^{*}(k) d k .
$$

Proof. (1) Since $R(\zeta)$ is permutable with $L$, the second relation of (5.31) is immediate from (5.5). On the other hand, we have in virtue of Lemma 5.5

$$
E(e)^{2}=W_{ \pm}(e) Z_{ \pm}(e) W_{ \pm}(e) Z_{ \pm}(e)=W_{ \pm}(e) E_{0}(e) Z_{ \pm}(e),
$$

where (5.17) and (5.20) show that $E_{0}(e) Z_{ \pm}(e)=Z_{ \pm}(e)$. Hence we have the first equality of (5.31).

(2) For $f, g$ in $C_{0}^{\infty}\left(E_{3}\right)$, it follows from (5.10) and (5.12) that

$$
\begin{aligned}
& \left(R(\zeta) E_{0}(e) f, g\right)-\left(R(\zeta) W_{ \pm}(e) f, g\right)=\left(X_{ \pm}(e) f, R(\zeta)^{*} g\right) \\
& =\lim _{\varepsilon \rightarrow+0} \frac{1}{2 \pi i} \int_{e} \frac{1}{\lambda \mp i \varepsilon-\zeta}\left(\left\{R_{0}(\lambda+i \varepsilon)-B_{0}(\lambda-i \varepsilon)\right\} f, V^{*}\left\{R(\lambda \mp i \varepsilon)^{*}\right.\right. \\
& =\int_{K_{e}} \frac{1}{|k|^{2}-\zeta} f(k)\left\{\overline{\hat{g}(k)}-\overline{\hat{g}_{\tau}(k)}-\overline{\left.\left[V^{*} R(\zeta)^{*} g\right]^{\wedge}(k)\right\}} d k\right. \\
& =\left(R_{0}(\zeta) E_{0}(e) f, g\right)-\left(W_{ \pm}(e) R_{0}(\zeta) f, g\right)-\left(R_{0}(\zeta) E_{0}(e) f, V^{*} R(\zeta)^{*} g\right) \\
& =\left(R(\zeta) E_{0}(e) f, g\right)-\left(W_{ \pm}(e) R_{0}(\zeta) f, g\right),
\end{aligned}
$$

where we have made use of the Parseval equality, relation (5.24) and the resolvent equation (1.6). Thus we have

$$
\left(R(\zeta) W_{ \pm}(e) f, g\right)=\left(W_{ \pm}(e) R_{0}(\zeta) f, g\right)
$$

proving the first equality of (5.32). Applying $Z_{ \pm}(e)$ to the first equality from the both sides and noting the relation $Z_{ \pm}(e)=E_{0}(e) Z_{ \pm}(e)$ 
$=Z_{ \pm}(e) E(e)$, we get the second equality. (5.33) is immediate from (5.32). Finally, since $\mathscr{D}(L)=\mathscr{D}\left(L_{0}\right),(5.32)$ implies that $W_{ \pm}(e) f$ and $Z_{ \pm}(e) f$ are both in $\mathscr{D}(L)$ if $f \in \mathscr{D}(L)$. Hence, by $(5$. 25) we see that $E(e) f \in \mathscr{D}(L)$ if $f \in \mathscr{D}(L)$. This proves (5.34).

(3) This statement is already proved implicitly in Lemma 5.5. For (5.25) and (5.26) show that $W_{ \pm}(e)$ maps $E_{0}(e)$ S one-to-one onto $E(e) \mathfrak{S}$, while $Z_{ \pm}(e)$ maps $E(e) \mathfrak{S}$ one-to-one onto $E_{0}(e) \mathfrak{S}$. Thus, if we note that the Fourier transformation gives a unitary map of $E_{0}(e) \mathfrak{E}$ onto $L^{2}\left(K_{e}\right), f_{ \pm}^{*}$ is nothing but $\left[Z_{ \pm}(e) f\right]^{\wedge}(k)$ and (5.36) follows from (5. 16) and (5.25).

(4) For $e=(\alpha, \beta) \in \Gamma$ we put $e_{\lambda}=(\alpha, \lambda), \alpha<\lambda<\beta$, and $E_{\alpha, \lambda}$ $=E\left(e_{\lambda}\right)$. Obviosly $E_{\gamma, \beta}=E(e)$. Let $f(x)$ be in $E(e) \mathscr{D}(L)$. Then we have from (5.33) that for any $g$ in $\mathfrak{g}$

$$
\begin{aligned}
& (L f, g)=(L E(e) f, g)=\left(W_{ \pm}(e) L_{0} E_{0}(e) Z_{ \pm}(e) f, g\right) \\
& \quad=\int_{e} \lambda d\left(E_{0, \lambda} Z_{ \pm}(e) f, W_{ \pm}(e)^{*} g\right)=\int_{e} \lambda d\left(E_{\kappa, \lambda} f, g\right) .
\end{aligned}
$$

Thus we have (5.35) taking (5.36) into account. The domain characterization of $L$ is immediate from representation (5.37) of $L$.

Q.E.D.

Remark 5.1. We may replace $E(e), \varphi_{ \pm}^{*}(x, k)$ and $\mathcal{P}_{x}(x, k)$ by $E^{*}(e), \varphi_{ \pm}(x, k)$ and $\varphi_{x}^{*}(x, k)$, respectively, in the above statements in order to get similar results for $L^{*}$, where $E^{*}(e)$ is defined by

$$
\left(E^{*}(e) f, g\right)=\lim _{\varepsilon \rightarrow+0} \frac{1}{2 \pi i} \int_{e}\left(\left\{R^{*}(\lambda+i \varepsilon)-R^{*}(\lambda-i \varepsilon)\right\} f, g\right) d \lambda
$$

for each $e$ in $\Gamma$. It is easy to verify that

$$
E^{*}(e)=E(e)^{*} .
$$

Remark 5.2. If $q(x)$ is assumed to satisfy in addition to (A) the smallness condition (2.15), then the spectral projection $E(e)$ can be constructed for any subinterval of $(-\infty, \infty)$. Put $E_{\lambda}=E\left(e_{\lambda}\right)$, where $e_{\lambda}=(-\infty, \lambda)$. Then the following relations hold.

$$
E_{\infty}=I, \quad L=\int_{0}^{\infty} \lambda d E_{\lambda} .
$$


Proof. We showed in Remark 2.1 that under condition (2.15), $\Sigma$ is empty, that is, $(-\infty, \infty) \in \widetilde{\Gamma}_{+} \cap \widetilde{\Gamma}_{-}$. Hence by $(5.3)_{ \pm}$we have

$$
\int_{-\infty}^{\infty}\left\{\|A R(\lambda+i \varepsilon) f\|^{2}+\|A R(\lambda-i \varepsilon) f\|^{2}\right\} d \lambda \leq C_{A}\|f\|^{2}, \quad \varepsilon \geq 0,
$$

for each $f$ in $\mathfrak{S}$. This and (5.2) imply that $A R(\zeta) f$ and $A R_{0}(\zeta) f$ both belong to the Hardy class $\mathcal{H}^{2}\left(\Omega_{ \pm} ; \mathfrak{S}\right),{ }^{16)}$ where $\Omega_{ \pm}=\{\zeta ; \operatorname{Im} \zeta \gtrless 0\}$, for each $f$ in $\mathfrak{S}$. Since $E_{0}((-\infty, \infty))=I$, we have from (5.8)

$$
\begin{aligned}
\left(E_{\propto} f, g\right)=(f, g) & -\frac{1}{2 \pi i} \int_{-\infty}^{\infty}\left(A R(\lambda+i 0) f, B^{*} R_{0}(\lambda-i 0) g\right) d \lambda \\
& +\frac{1}{2 \pi i} \int_{-\infty}^{\infty}\left(A R(\lambda-i 0) f, B^{*} R_{0}(\lambda+i 0) g\right) d \lambda .
\end{aligned}
$$

$A R(\lambda \pm i 0) f$ and $B^{*} R_{0}(\lambda \mp i 0) g$ are boundary functions of the class $\mathcal{H}^{2}\left(\Omega_{ \pm} ; \mathfrak{S}\right)$ and $\mathcal{H}^{2}\left(\Omega_{\mp} ; \mathfrak{S}\right)$, respectively. Hence, both the second and the third terms in the right member of (5.42) are zero, and the first relation of $(5.41)$ is proved. The second relation is obvious from (5.38).

Q.E.D.

\section{Scattering Theory}

\section{$\S 6$. Wave operators.}

In this $\S$ we shall develop the time dependent scattering theory restricting $L_{0}$ and $L$ to the invariant subspaces $E_{0}(e) \mathfrak{S}$ and $E(e) \mathfrak{S}$, respectively, where $e$ may be any interval in $\Gamma$.

We set $\mathfrak{S}_{0}(e)=E_{0}(e) \mathfrak{g}$ and $\mathfrak{S}(e)=E(e) \mathfrak{L}$. Then $\mathfrak{S}_{0}(e)$ is a Hibert space with respect to the $L^{2}$-norm, and $\mathfrak{g}(e)$ is a Banach space with the dual space $\mathfrak{S}^{*}(e)=E^{*}(e) \mathfrak{S}$, where $E^{*}(e)=E(e)^{*}$. Put

$$
L_{0}(e)=L_{0} E_{0}(e), \quad L(e)=L E(e) .
$$

Then $L_{0}(e)$ is a selfadjoint operator in $\mathfrak{S}_{0}(e)$ with the domain $E_{0}(e) \mathscr{D}\left(L_{0}\right)$ and generates the unitary group $\exp \left\{-i t L_{0}(e)\right\}$ $=\exp \left\{-i t L_{0}\right\} E_{0}(e)(-\infty<t<\infty)$, while $L(e)$ is a closed operator in $\mathfrak{S}(e)$ with the domain $E(e) \mathscr{D}(L)=E(e) \mathscr{D}\left(L_{0}\right)$. Note that $L(e)$ has

16) See Kato [13]; Remark 1.4. 
purely the continuous spectrum which fills the closure of $e$. Let us consider the operator $U_{ \pm}(t ; e)$ defined in $\mathfrak{S}(e)$ by

$$
U_{ \pm}(t ; e)=W_{ \pm}(e)\left\{-i t L_{0}\right\} Z_{ \pm}(e), \quad-\infty<t<\infty .
$$

It is obvious that $\left\{U_{ \pm}(t ; e)\right\}$ forms a strongly continuous group of type zero.

Moreover, we have the

Lemma 6.1. $-i L(e)$ is the infinitesimal generator of the group $\left\{U_{ \pm}(t ; e)\right\}$ :

$$
\exp \{-i t L(e)\}=U_{1}(t ; e)=U_{-}(t ; e), \quad-\infty<t<\infty .
$$

Proof. We have only to show that $R(\zeta ; e)=(L(e)-\zeta I)^{-1}=R(\zeta) E(e)$ is the Laplace transform of $U_{ \pm}(t ; e)$. For example, we assume $\operatorname{Im} \zeta>0$. Then for each $f \in \mathfrak{S}(e)$ and $g \in \mathfrak{S}$, we have, using the Parseval equality

$$
\begin{aligned}
& i \int_{0}^{\infty} \exp \{i \zeta t\}\left(U_{ \pm}(t ; e) f, g\right) d t \\
= & i \int_{0}^{\infty} \exp \{i \zeta t\} d t \int_{K_{e}} \exp \left\{-i t|k|^{2}\right\}\left[Z_{ \pm}(e) f\right]^{\wedge}(k)\left[\overline{\left.W_{ \pm}(e)^{*} g\right]^{\wedge}(k)} d k\right. \\
= & \int_{K_{e}} \frac{1}{|k|^{2}-\zeta}\left[Z_{ \pm}(e) f\right]^{\wedge}(k)\left[\overline{\left.W_{ \pm}(e)^{*} g\right]^{\wedge}(k)} d k\right. \\
= & \left(W_{ \pm}(e) R_{0}(\zeta) Z_{ \pm}(e) f, g\right)=(R(\zeta) E(e) f, g)
\end{aligned}
$$

by (5.25) and (5.32). This implies that

(6. 4) $\quad R(\zeta ; e)=i \int_{0}^{\infty} \exp \{i \zeta t\} U_{ \pm}(t ; e) d t \quad$ if $\quad \operatorname{Im} \zeta>0$.

Similarly we have

$$
R(\zeta ; e)=-i \int_{-\infty}^{0} \exp \{i \zeta t\} U_{ \pm}(t ; e) d t \quad \text { if } \quad \operatorname{Im} \zeta<0 .
$$

Thus, (6.3) is proved.

Q.E.D.

Remark 6. 1. We put $L^{*}(e)=L^{*} E^{*}(e)$. Then $L^{*}(e)$ is a closed operator in $\mathfrak{S}^{*}(e)$ with domain $E^{*}(e) \mathscr{D}\left(L^{*}\right)=E(e) * \mathscr{D}(L)$, and

$$
L *(e)=L(e)^{*} .
$$


Thus, it follows that $-i L^{*}(e)$ is the infinitesimal generator of the group

$$
\exp \left\{-i t L^{*}(e)\right\}=\left(\exp ^{*}\{i t L(e)\}\right)^{*}, \quad-\infty<t<\infty .
$$

Lemma 6. 2. Put $a(x)=a_{1}(x)=|q(x)|^{1 / 2}$. Then $A R(\zeta ; e) f=A(L(e)$ $-\zeta I)^{-1} f$ and $A R^{*}(\zeta ; e) g=A\left(L^{*}(e)-\zeta I\right)^{-1} g$ both belong to the Hardy class $\mathscr{H}^{2}\left(\Omega_{ \pm} ; \mathfrak{E}\right)$ for each $f \in \mathfrak{E}(e)$ and $g \in \mathfrak{E} *(e)$ :

(6. 9) $\int_{-\infty}^{\infty}\left\{\left\|A R^{*}(\lambda+i \varepsilon ; e) g\right\|^{2}+\left\|A R^{*}(\lambda-i \varepsilon ; e) g\right\|^{2}\right\} d \lambda \leq C_{A, e}\|g\|^{2}$, with $C_{A, e}>0$ independent of $\varepsilon>0$.

Proof. Given any $e=(\alpha, \beta) \in \Gamma$ and $f \in \mathfrak{Q}(e), A R(\zeta ; e) f=A R(\zeta) f$ $(\sqrt{\zeta} \notin \Sigma)$ and is an $\mathfrak{S}$-valued analytic function of $\zeta$ outside $(\alpha, \beta)$, and $\| A R(\zeta ; e) f i \mid \leq$ const $\|f\|$ uniformly in $\operatorname{Re} \zeta \notin(\alpha-\delta, \beta+\delta)$ for a fixed $\delta>0$. We choose $\delta>0$ small and $N>0$ large such that $(\alpha-\delta, \beta+\delta),(-\infty,-N)$ and $(N, \infty)$ all belong to the class $\widetilde{\Gamma}_{+} \cap \widetilde{\Gamma}_{-}$. Then we have

$$
\int_{-\infty}^{-N}+\int_{\omega-\delta}^{\beta+\delta}+\int_{N}^{\infty}\|A R(\lambda \pm i \varepsilon ; e) f\|_{\mid}^{2} d \lambda \leq C_{A, e}\|f\|^{2}, \quad \text { for } \varepsilon>0
$$

by Lemma 5.1 . Thus, we get (6.8). (6.9) can be proved by the same reasoning.

Q.E.D.

Using this lemma, we can now prove the

Lemma 6. 3. Put $a(x)=a_{1}(x)$. Then we have for arbitrary $f, g$ in $\mathfrak{S}$

$$
\begin{aligned}
& \left(W_{ \pm}(e) f, g\right)=\left(E_{0}(e) f, E^{*}(e) g\right) \\
& \quad \mp \frac{1}{2 \pi i} \int_{-\infty}^{\infty}\left(A R_{0}(\lambda \pm i 0) E_{0}(e) f, B^{*} R^{*}(\lambda \pm i 0 ; e) E^{*}(e) g\right) d \lambda,
\end{aligned}
$$

$$
\begin{aligned}
& \left(Z_{ \pm}(e) f, g\right)=\left(E(e) f, E_{0}(e) g\right) \\
& \quad \pm \frac{1}{2 \pi i} \int_{-\infty}^{\infty}\left(B R(\lambda \pm i 0 ; e) E(e) f, A^{*} R_{0}(\lambda \pm i 0) E_{0}(e) g\right) d \lambda .
\end{aligned}
$$

Proof. Since both $A R_{0}(\zeta) E_{0}(e) f$ and $B^{*} R^{*}(\zeta ; e) E^{*}(e) g$ are in the Hardy class $\mathcal{H}^{2}\left(\Omega_{ \pm} ; \mathfrak{F}\right)$ for each $f, g$ in $\mathfrak{g}$, it follows from (5.10) that 


$$
\begin{aligned}
& \left(X_{ \pm}(e) E_{0}(e) f, E^{*}(e) g\right) \\
& \quad=\frac{1}{2 \pi i} \int_{-\infty}^{\infty}\left(A\left\{R_{0}(\lambda+i 0)-R_{0}(\lambda-i 0)\right\} E_{0}(e) f, B^{*} R^{*}(\lambda \pm i 0 ; e) E^{*}(e) g\right) d \lambda \\
& \quad= \pm \frac{1}{2 \pi i} \int_{-\infty}^{\infty}\left(A R_{0}(\lambda \pm i 0) E_{0}(e) f, B^{*} R^{*}(\lambda \pm i 0 ; e) E^{*}(e) g\right) d \lambda .
\end{aligned}
$$

With the relation $W_{ \pm}(e)=E(e) W_{ \pm}(e) E_{0}(e)$ and $(5.12)$, this proves (6.10). (6.11) can be proved by the same reasoning. Q.E.D.

The above representations of $W_{ \pm}(e)$ and $Z_{ \pm}(e)$ enable us to follow the same way as Kato [13] to obtain the following theorem.

Theorem 6.1. $W_{ \pm}(e)$ and $Z_{ \pm}(e)$ satisfy the following relations:

$$
\begin{aligned}
& W_{ \pm}(e)=\operatorname{s}_{t \rightarrow \pm \infty} \exp \{i t L(e)\} E(e) \exp \left\{-i t L_{0}(e)\right\}, \\
& Z_{ \pm}(e)=\underset{t \rightarrow \pm \infty}{\mathrm{s}-\lim _{t \rightarrow \infty}} \exp \left\{i t L_{0}(e)\right\} E_{0}(e) \exp \{-i t L(e)\} \text {. }
\end{aligned}
$$

Hence, $W_{ \pm}(e)$ are the wave operators for the pair $L_{0}(e), L(e)$ in the time dependent formulation, and $Z_{ \pm}(e)$ are the inverse wave operators.

Proof. (see [13]; Theorem 3.9) We sketch a proof for $W_{\perp}(e)$. Replace in (6.10) $f$ and $g$ respectively by $\exp \left\{-i s L_{0}(e)\right\} f\left(f \in \mathfrak{S}_{0}(e)\right)$ and $\exp \left\{-i s L^{*}(e)\right\} E^{*}(e) g(g \in \mathfrak{S})$. Then we have, using (6.7) and the Parseval equality,

$$
\begin{aligned}
\left(W_{1}(e) f, g\right) & =\left(\exp \{i s L(e)\} E(e)\left\{-i s L_{0}(e)\right\} f, g\right) \\
& -\int_{s}^{\infty}\left(A \exp \left\{-i t L_{0}(e)\right\} f, B^{*} \exp \left\{-i t L^{*}(e)\right\} E^{*}(e) g\right) d t
\end{aligned}
$$

Thus, we obtain

$$
\begin{aligned}
& \left|\left(W_{+}(e) f-\exp \{i s L(e)\} E(e) \exp \left\{-i s L_{0}(e)\right\} f, g\right)\right| \\
& \leq\left[\int_{s}^{\infty}\left\|A \exp \left\{-i t L_{0}(e)\right\} f\right\|^{2} d t\right]^{1 / 2}\left[\int_{0}^{\infty}\left\|B^{*} \exp \left\{-i t L^{*}(e)\right\} E^{*}(e) g\right\|^{2} d t\right]^{1 / 2} \\
& \leq \mathrm{const}\left[\int_{s}^{\infty}\left\|A \exp \left\{-i t L_{0}(e)\right\} f^{2}\right\|^{2} d t\right]^{1 / 2}|| E^{*}(e) g \| .
\end{aligned}
$$

Since $\left\|E^{*}(e) g\right\| \leq \mathrm{const}\|g\|$, this proves that $\exp \{i s L(e)\} E(e) \exp$ $\left\{-i s L_{0}(e)\right\}$ strongly converges as $s \rightarrow+\infty$ to $W_{+}(e)$ Q. E. D.

Remark 6.2. For each $f \in \mathfrak{S}_{0}(e), \exp \{-i t L(e)\} f$ gives a solution of the initial value problem 


$$
i \frac{\partial}{\partial t} u(t)=L u(t), \quad u(0)=f \quad(-\infty<t<\infty) .
$$

It should be, however, noted that solutions in $\mathscr{E}$ of (6.14) are not always unique. If we assume on $q(x)$ in addition to (A) the condition that

$$
\operatorname{Im}[q(x)] \text { is bound in } x \in E_{3},
$$

then $-i L$ is known to be the infinitesimal generator in $\mathfrak{S}$ of the group $\exp \{-i t L\}$. Hence, in this case $\exp \{-i t L(e)\} f=\exp \{-i t L\} f$ is a unique solution of (6.14). On the other hand, if $q(x)$ is assumed to satisfy in addition to (A) the "smallness condition" (2.15), then $L$ with such a $q(x)$ also becomes the infinitesimal generator in $\mathfrak{S}$ of the group $\exp \{-i t L\}=W_{ \pm} \exp \left\{-i t L_{0}\right\} Z_{ \pm}$, where $W_{ \pm}=W_{ \pm}((0, \infty))$ and $Z_{ \pm}=Z_{ \pm}((0, \infty))$, since we proved in Remark 5.2 that $E((-\infty, \infty))$ $=E((0, \infty))=I$. So, the above condition (6.15) is not necessary to verify that $L$ is the infinitesimal generator of a strongly continuous group.

Now we see from Lemma 6.1 that the initial value problem

$$
i \frac{\partial}{\partial t} u(t)=L(e) u(t), \quad u(0)=f \in \mathfrak{S}(e)
$$

has a unique solution $u(t)=\exp \{-i t L(e)\} f$ in $\mathfrak{S}(e)$. From the above theorem, it follows that the solution satisfies the following asymptotic conditions :

$$
\lim _{t \rightarrow \pm \infty}\left\|u(t)-\exp \left\{-i t L_{0}(e)\right\} f_{ \pm}\right\|=0
$$

where

$$
f_{ \pm}=Z_{ \pm}(e) f\left(\in \mathfrak{S}_{0}(e)\right) .
$$

The scattering operator $S(e)$ acting in $\mathfrak{S}_{0}(e)$ can be defined by

$$
S(e)=Z_{+}(e) W_{-}(e)=W_{+}(e)^{-1} W_{-}(e):
$$

Theorem 6. 2. $S(e)$ is permutable with $L_{0}(e)$, i.e.,

$$
S(e) L_{0}(e) \subset L_{0}(e) S(e),
$$

and is a one-to-one mapping of $\mathfrak{S}_{0}(e)$ onto itself with the inverse 
$S(e)^{-1}=Z_{-}(e) W_{+}(e)$. Moreover, $f_{+}$and $f_{-}$defined by (6.18) are related to each other by the equality

$$
f_{+}=S(e) f_{-} .
$$

Proof. The proof of this theorem is immediate from (5.25) and (5.26).

Q. E. D.

Remark 6. 3. If $q(x)$ satisfy the conditions given in Remark 2. 3: $\operatorname{Im}[q(x)] \leq 0$ and $\operatorname{Re}[q(x)]$ is a small perturbation in the sense of Remark 2.1, then both $Z_{+}=Z_{+}((0, \infty))$ and $W_{-}=W_{-}((0, \infty))$ exist, and the solution $u(t)$ for $t \geq 0$ of the initial value problem

$$
i \frac{\partial}{\partial t} u(t)=L u(t), \quad u(0)=W_{-} f(f \in \mathfrak{E})
$$

is unique and satisfies the following asymptotic condition :

$$
\lim _{t \rightarrow \infty}\left\|u(t)-\exp \left\{-i t L_{0}\right\} S f\right\|=0, \quad S=Z_{+} W_{-} .
$$

Proof. We proved in Remark 2.3 that with our condition on $q(x)$ the set $\Sigma$ is contained in $\{\kappa ; \operatorname{Re} \kappa<0, \operatorname{Im} \kappa \geqslant 0\}$, i.e., $(0, \infty) \in \Gamma_{+}$. Hence $W_{-}$and $Z_{+}$both exist. Moreover, the "dissipativity condition" $\operatorname{Im}[q(x)] \leq 0$ shows that $-i L$ is the infinitesimal generator of the semi group $\exp \{-i t L\}(t \geq 0)$. Hence, the solution for $t \geq 0$ of (6.22) is unique and is given by

$$
u(t)=\exp \{-i t L\} u(0)=\exp \{-i t L\} W_{-} f .
$$

Applying $\exp \left\{i t L_{0}\right\}$ to this from the left, and noting relation (6.13), we have

$$
\exp \left\{i t L_{0}\right\} u(t)-Z_{+} W_{-} f \rightarrow 0 \quad(\text { as } t \rightarrow \infty)
$$

which proves (6.23) since exp $\left\{i t L_{0}\right\}$ forms a unitary group. Q. E. D.

$\S 7$. Uniqueness of solutions for the scattering inverse problem.

In this $\S$ we require the following stronger assumption on $q(x)$ :

$$
(1+|x|)^{(3+\delta) / 2} q(x) \in L^{2}\left(E_{3}\right), \quad \delta>0 .
$$

With this assumption on $q(x)$, we can proceed along the same line 
of proof as given by Povzner ([2]; II, Lemma 1) or Ikebe ([5]; Lemma 4.2$)$ to derive that the distorted plane waves $\varphi_{ \pm}(x, k)$, $\mp|k| \notin \Sigma$, constructed in $\S 5$ have the following asymptotic expansions for large $|x|:{ }^{17}$

$$
\begin{aligned}
\varphi_{ \pm}(x, k)=\exp \{i k \cdot x\} & +\frac{\exp \{\mp i|k| \cdot|x|\}}{|x|} \theta_{ \pm}(n, \nu ;|k|) \\
& +0\left(|x|^{-1-\gamma}\right) ;
\end{aligned}
$$

$$
\theta_{ \pm}(n, \nu ;|k|)=-\frac{1}{4 \pi} \int_{E_{3}} \varphi_{ \pm}(y,|k| \nu) \exp \{ \pm i|k| n \cdot y\} d y
$$

where $n=x /|x|, \nu=k /|k|$ and $\gamma=\delta / 2(2+\delta)$.

The purpose of this $\S$ is to represent the scattering operator $S(e)$ given by (6.19) explicitly in terms of the scattering amplitude $\theta_{-}(n, \nu ; k)$, and then to derive that the potential function $q(x)$ is uniquely determined from a given function $\theta_{-}(n, \nu ; k)$ if it is assumed to satisfy assumption $\left(\mathrm{A}_{1}\right)$.

We begin with showing the symmetricity in $n$ and $\nu$ of $\theta_{ \pm}(n, \nu ;|k|)$. It follows from (4.15) that

$$
\overline{\varphi_{ \pm}(x,-k)}=\exp \{i k \cdot x\}-\int_{E_{3}} \frac{\exp \{ \pm i|k| \cdot|x-y|\}}{4 \pi|x-y|} \overline{q(y) \phi_{ \pm}(y,-k)} d y .
$$

On the other hand, we see from (2) of Lemma 4.2 that this equation has a unique solution $\phi_{*}^{*}(x, k)$. Hence we have

$$
\varphi_{\mp}^{*}(x, k)=\overline{\varphi_{ \pm}(x,-k)} .
$$

Note that each $\varphi_{\mp}^{*}(x, k)$ also has the asymptotic form (7.1) with $\theta_{ \pm}(n, \nu ;|k|)$ replaced by

$$
\theta_{ \pm}^{*}(n, \nu ;|k|)=\overline{\theta_{\mp}(n,-\nu ;|k|)} .
$$

Moreover, we have the following

Lemma 7.1. $\theta_{ \pm}(n, \nu ;|k|)$ satisfy the equalities

$$
\theta_{+}(n, \nu ;|k|)=\theta_{+}(\nu, n ;|k|) \text {, }
$$

17) In [5], $q(x)$ is assumed to be in $L_{\text {loc }}^{2}\left(E_{3}\right)$ and behave like $0(|x|)^{-3-\delta)}(\delta>0)$ at infinity in order to obtain this expansion with $\gamma=\delta / 2$. In our case, however, the use of the Schwarz inequality makes $r$ as given above. 


$$
\theta_{-}(n, \nu ;|k|)=\theta_{-}(-\nu,-n ;|k|) .
$$

Proof. Put $k^{\prime}=|k| n$. Then we have from (4.15) and (7.2)

$$
\begin{aligned}
& \theta_{+}(n, \nu ;|k|)=-\frac{1}{4 \pi} \int_{E_{3}} \varphi_{+}(y, k) q(y) \exp \left\{i k^{\prime} \cdot y\right\} d y \\
& =-\frac{1}{4 \pi} \int_{E_{3}} \varphi_{+}(y, k) q(y)\left[\varphi_{+}\left(y, k^{\prime}\right)+\int_{E_{3}} \frac{\exp \left\{-i\left|k^{\prime}\right| \cdot|y-z|\right\}}{4 \pi|y-z|}\right. \\
& \left.\times q(z) \varphi_{+}\left(z, k^{\prime}\right) d z\right] d y .
\end{aligned}
$$

Since $q(z) \in L^{1}\left(E_{3}\right)$ by assumption $\left(\mathrm{A}_{1}\right)$, we can exchange the order of the integrations to obtain

$$
\theta_{\uparrow}(n, \nu ;|k|)=-\frac{1}{4 \pi} \int_{E_{3}} \exp \{i k \cdot z\} q(z) \varphi_{\dashv}\left(z, k^{\prime}\right) d z=\theta_{\vdash}(\nu, n ;|k|)
$$

noting $\left|k^{\prime}\right|=|k|$. This proves (7.5). We have similarly equality (7.5) for $\theta_{+}^{*}(n, \nu ; k)$. Then it follows from (7.4) that

$$
\theta_{-}(n, \nu ;|k|)=\overline{\theta_{+}^{*}(n,-\nu ;|k|)}=\overline{\theta_{+}^{*}(-\nu, n ;|k|)}=\theta_{-}(-\nu,-n ;|k|)
$$

which proves (7.6).

Q. E.D.

We can now prove the following theorem :

Theorem 7.1. ${ }^{18)}$ For each $e \in \Gamma$ and $f \in \mathcal{E}_{0}(e)$, the scattering operator $S(e)$ is given the following representation in the Fourier space :

$$
\begin{aligned}
& {[S(e) f]^{\wedge}(|k| \nu)=\hat{f}(|k| \nu)+\frac{i}{2 \pi} \int_{|n|=1}|k| \theta_{-}(-n,-\nu ;|k|) \hat{f}(|k| n) d n} \\
& =\hat{f}(|k| \nu)+\frac{i}{2 \pi} \int_{|n|=1} \mid k \overline{\mid \theta_{+}^{*}(-n, \nu ;|k|)} \hat{f}(|k| n) d n, \\
& |k|^{2} \in e \text {. }
\end{aligned}
$$

Proof. Since $W_{-}(e)=E(e) W_{-}(e)=W_{+}(e) S(e)$ by (5.25) and (6.19), we have, taking (5.16) into account,

$$
\begin{aligned}
& {\left[W_{+}(e)\{S(e)-I\} f\right](x)=\left[\left\{W_{-}(e)-W_{+}(e)\right\} f\right](x)} \\
& \quad=(2 \pi)^{-3 / 2} \int_{K_{e}}\left\{\varphi_{-}(x, k)-\varphi_{+}(x, k)\right\} \hat{f}(k) d k, \quad f \in \mathfrak{S}_{0}(e) .
\end{aligned}
$$

It follows from (4.13) and (4.17) that

18) Cf. Ikebe [6]; Theorem 1. 


$$
\begin{aligned}
& \varphi_{-}(x, k)-\varphi_{+}(x, k) \\
& =-\int_{E_{3}} \frac{\exp \{i|k| \cdot|x-y|\}}{4 \pi|x-y|} b_{2}(y) \psi_{-}(y, k) d y \\
& \quad+\int_{E_{3}} \frac{\exp \{-i|k| \cdot|x-y|\}}{4 \pi|x-y|} b_{2}(y) \psi_{+}(y, k) d y \\
& =-\int_{E_{3}} \frac{\exp \{i|k| \cdot|x-y|\}-\exp \{-i|k| \cdot|x-y|\}}{4 \pi|x-y|} q(y) \varphi_{-}(y, k) d y \\
& \quad+\int_{E_{3}} \frac{\exp \{-i|k| \cdot|x-y|\}}{4 \pi|x-y|} b_{2}(y)\left\{\psi_{+}(y, k)-\psi_{-}(y, k)\right\} d y \\
& =J_{1}+J_{2},
\end{aligned}
$$

where $\psi_{ \pm}(x, k)=a_{2}(x) \varphi_{ \pm}(x, k) \in L^{2}\left(E_{3}\right)$ as a function of $x$, and

$$
\left[\left\{I+Q_{0}(\mp|k|)\right\} \psi_{ \pm}\right](x, k)=a_{2}(x) \exp \{i k \cdot x\} .
$$

Put $k^{\prime}=|k| \nu,|\nu|=1$, and note the relation

$$
\text { (7.10) } \begin{aligned}
\int_{|\nu|=1} & \exp \left\{i k^{\prime} \cdot x\right\} d \nu \\
= & \frac{2 \pi}{i|k| \cdot|x|}\{\exp \{i|k| \cdot|x|\}-\exp \{-i|k| \cdot|x|\}\} .
\end{aligned}
$$

Then

$$
\begin{aligned}
J_{1} & =-\frac{i|k|}{8 \pi^{2}} \int_{|\nu|=1} \exp \left\{i k^{\prime} \cdot x\right\} d \nu \int_{E_{3}} q(y) \varphi_{-}(y, k) \exp \{-i|k| \nu \cdot y\} d y \\
& =\frac{i|k|}{2 \pi} \int_{\mid \nu_{i}=1} \theta_{-}(\nu, n ;|k|) \exp \left\{i k^{\prime} \cdot x\right\} d \nu
\end{aligned}
$$

where we put $k=|k| n$. On the other hand, it follows from (7.9) and (7.10) that

$$
\begin{aligned}
& {\left[\left\{I+Q_{0}(-|k|)\right\}\left(\psi_{-}-\psi_{-}\right)\right](x, k)} \\
& \quad=a_{2}(x) \exp \{i k \cdot x\}-\left[\left\{I+Q_{0}(-|k|)\right\} \psi_{-}\right](x, k) \\
& \quad=\left[\left\{Q_{0}(|k|)-Q_{0}(-|k|)\right\} \psi_{-}\right](x, k) \\
& \quad=a_{2}(x) \int_{E_{3}} \frac{\exp \{i|k| \cdot|x-y|\}-\exp \{-i|k| \cdot|x-y|\}}{4 \pi|x-y|} q(y) \mathcal{P}_{-}(y, k) d y \\
& \quad=\frac{i|k|}{8 \pi^{2}} \int_{|\nu|=1} a_{2}(x) \exp \left\{i k^{\prime} \cdot x\right\} d \nu \int_{E_{3}} q(y) \varphi_{-}(y, k) \exp \{-i|k| \nu \cdot y\} d y \\
& \quad=-\frac{i|k|}{2 \pi} \int_{|\nu|=1}\left[\left\{I+Q_{0}\left(-\left|k^{\prime}\right|\right)\right\} \psi_{+}\right]\left(x, k^{\prime}\right) \theta_{-}(\nu, n ;|k|) d \nu .
\end{aligned}
$$


Since $\left|k^{\prime}\right|=|k|$ and $I+Q_{0}(-|k|)$ is invertible in $\mathscr{S}$ except for $-|k| \in \Sigma$, this shows that

$$
\psi_{+}(x, k)-\psi_{-}(x, k)=-\frac{i|k|}{2 \pi} \int_{|\nu|=1} \theta_{-}(\nu, n ;|k|) \psi_{+}\left(x, k^{\prime}\right) d \nu .
$$

Summing up we have now

$$
\begin{gathered}
\varphi_{+}(x, k)-\varphi_{-}(x, k)=\frac{i|k|}{2 \pi} \int_{|\nu|=1} \theta_{-}(\nu, n ;|k|)\left[\exp \left\{i k^{\prime} \cdot x\right\}-\right. \\
\left.\quad-\int_{E_{3}} \frac{\exp \left\{-i\left|k^{\prime}\right| \cdot|x-y|\right\}}{4 \pi|x-y|} b_{2}(y) \psi_{+}\left(y, k^{\prime}\right) d y\right] d \nu \\
=\frac{i|k|}{2 \pi} \int_{|\nu|=1} \theta_{-}(\nu, n ;|k|) \varphi_{+}\left(x, k^{\prime}\right) d \nu .
\end{gathered}
$$

Substitute this in (7.8). Then

$$
\begin{aligned}
& {\left[W_{+}(e)\{S(e)-I\} f\right](x)=(2 \pi)^{-3 / 2} \int_{K_{e}} \varphi_{+}(x, k)[\{S(e)-I\} f]^{\wedge}(k) d k} \\
& \quad=(2 \pi)^{-3 / 2} \frac{i}{2 \pi} \int_{e}|k|^{3} d|k| \int_{|n|=1} \hat{f}(|k| n) d n \int_{|\nu|=1} \theta_{-}(\nu, n ;|k|) \varphi_{+}(x,|k| \nu) d \nu \\
& =(2 \pi)^{-3 / 2} \frac{i}{2 \pi} \int_{K_{e}} \varphi_{+}\left(x, k^{\prime}\right) d k^{\prime} \int_{|n|=1}|k| \theta_{-}(\nu, n ;|k|) \hat{f}(|k| n) d n, \quad k^{\prime}=|k| \nu .
\end{aligned}
$$

This yields the representation (7.7) in virtue of (7.4) and (7.6) since the transform $\int_{K_{e}} \varphi_{+}(x, k) \hat{f}(k) d k$ gives a one-to-one mapping of $L^{2}\left(K_{e}\right)$ onto $\mathfrak{g}(e)$.

Q.E. D.

Remark 7.1. ${ }^{19)}$ If $q(x)$ is assumed to satisfy the stronger condition (2.17) in Remark 2.2, then the scattering operator can be continued analytically into $\operatorname{Im} \kappa>-\delta / 2$ and it is in this region a meromorphic function. The poles in $\operatorname{Im} \kappa>0$ correspond to the discrete eigenvalues of $L$ with $\zeta=\kappa^{2}$.

Proof. As we noted in Remark 2.2, $Q_{0}(\kappa)$ can be continued analytically into $\operatorname{Im} \kappa>-\delta / 2$ preserving the complete continuity in $\mathscr{S}$. Let $\widetilde{Q}_{0}(\kappa)$ denote the extended operator. Put $a(x)=|q(x)|^{1 / 2}$. Since

19) Cf. Lax-Phillips [21]. They developed a different method to get, among other things, a result concerning the analytic continuation of the scattering matrix under the assumption that $q(x)$ (real) is smooth and has a compact support (see Theorem 5.2). 
$|a(x)| \leq C \exp \{-(\delta / 2)|x|\}$ for large $|x|$, we see that $a(x) \exp \left\{i \kappa^{\prime} \nu \cdot x\right\}$, $|\nu|=1$, is in $\mathfrak{S}$ whenever $\operatorname{Im} \kappa^{\prime}>-\delta / 2$. Thus

$$
\widetilde{\psi}\left(x, \kappa^{\prime} \nu ; \kappa\right)=a(x) \exp \left\{i \kappa^{\prime} \nu \cdot x\right\}-\left[\widetilde{Q}_{0}(\kappa) \tilde{\psi}\right]\left(x, \kappa^{\prime} \nu ; \kappa\right)
$$

has a unique solution $\widetilde{\psi}\left(\cdot, \kappa^{\prime} \nu ; \kappa\right) \in \mathfrak{S}$ which depends analytically on $\kappa^{\prime}$ in $\operatorname{Im} \kappa^{\prime}>-\delta / 2$ and meromorphically on $\kappa$ in $\operatorname{Im} \kappa<-\delta / 2$. We put

$$
\begin{aligned}
& \widetilde{\theta}_{-}(n, \nu ; \kappa)=\frac{-1}{4 \pi} \int_{E_{3}} b(y) \widetilde{\psi}(y, \kappa \nu ; \kappa) \exp \{-i \kappa n \cdot y\} d y, \\
& \widetilde{\theta}_{+}(n, \nu ; \kappa)=\frac{-1}{4 \pi} \int_{E_{3}} b(y) \widetilde{\psi}(y, \kappa \nu ;-\bar{\kappa}) \exp \{i \kappa n \cdot y\} d y,
\end{aligned}
$$

The right members make sense for each $\kappa$ in $\operatorname{Im} \kappa>-\delta / 2$ except at the poles of $\left\{I+\widetilde{Q}_{0}(\kappa)\right\}^{-1}$ because $b(x) \exp \{i \kappa n \cdot x\} \in \mathcal{S}$ for such a $\kappa$. It is obvious that $\widetilde{\psi}(y,|k| \nu ;|k|)=\psi_{-}(y, k)$ and $\widetilde{\psi}(y,|k| \nu ;-|k|)=$ $\psi_{+}(y, k)$ with $\nu=k /|k|$. Thus, $\widetilde{\theta}_{ \pm}(n, \nu ; \kappa)$ defined by (7.12) gives an analytic continuation into $\operatorname{Im} \kappa>-\delta / 2$ of $\theta_{ \pm}(n, \nu ;|k|)$ which represents the scattering operator.

Next, let us prove that the potential $q(x)$ is uniquely determined by the asymptotic behavior for large $|k|$ of a given $\theta_{ \pm}(n, \nu ;|k|)$.

We choose $\alpha$ so large that the interval $(\alpha, \infty)$ is in $\Gamma$. It is obvious with assumption $\left(\mathrm{A}_{1}\right)$ that $\theta_{ \pm}(n, \nu ;|k|)$ is bounded and continuous in $n, \nu$ and $|k|^{2} \in[\alpha, \infty)$. In order to obtain an asymptotic representation for $|k| \rightarrow \infty$ of the function $\theta_{ \pm}(n, \nu ;|k|)$, we rewrite it as follows :

$$
\begin{aligned}
& \theta_{ \pm}(n, \nu ;|k|)=-\frac{1}{4 \pi} \int_{E_{3}} q(y) \exp \{i|k|( \pm n+\nu) \cdot y\} d y \\
& \quad+\frac{1}{4 \pi} \int_{E_{3}} q(y)\left[\int_{E_{3}} \frac{\exp \{\mp i|k| \cdot|y-z|\}}{4 \pi|y-z|}\right. \\
& \left.\quad \times q(z) \varphi_{ \pm}(z, k) d z\right] \exp \{ \pm i|k| n \cdot y\} d y
\end{aligned}
$$

taking (4.15) into account. For given and $\varepsilon>0$, we choose $q_{0}(x) \in$ $C_{0}^{\infty}\left(E_{3}\right)$ such that $\left\|q-q_{0}\right\|_{1+\delta} \leq \varepsilon$, where $\|\cdot\|_{1+\delta}$ is defined by (3.6) and put $q(x)=q_{0}(x)+\widetilde{q}(x)$. As is shown by Faddeev [4], we have $\int_{E_{3}} \frac{\exp \{\mp i|k| \cdot|y-z|\}}{4 \pi|y-z|} q_{0}(z) \varphi_{\dot{ \pm}}(z, k) d z=0\left(|k|^{-1}\right) \quad$ (uniformly in $\left.y\right)$. 
On the other hand, we have

$$
\begin{aligned}
& \sup _{y}\left|\int_{E_{3}} \frac{\exp \{\mp i|k| \cdot|y-z|\}}{4 \pi|y-z|} \widetilde{q}(z) \varphi_{ \pm}(z, k) d z\right| \\
& \quad \leq \text { const } \cdot \sup _{y}\left[\int_{E_{3}}|y-z|^{-2}(1+|z|)^{-1-\delta} d z\right]^{1 / 2}\|\widetilde{q}\|_{1+\delta} \leq \text { const } \cdot \varepsilon
\end{aligned}
$$

since $\varphi_{ \pm}(x, k)$ is bounded in $x \in E_{3}$ and $|k|^{2} \in[\alpha, \infty)$ (see Lemma 4.2). Thus, we have, noting that $q(x) \in L^{1}\left(E_{3}\right) \cap L^{2}\left(E_{3}\right)$,

$$
\begin{array}{r}
\theta_{ \pm}(n, \nu ;|k|)=-\frac{1}{4 \pi} \int_{E_{3}} q(y) \exp \{i|k|( \pm n+\nu) \cdot y\} d y+o(1), \\
\text { as }|k| \rightarrow \infty,
\end{array}
$$

uniformly in $n$ and $\nu$.

For an arbitrary vector $m \in E_{3}$ we can choose $|k|, n$ and $\nu$ so that $m=|k|(n \pm \nu)$. We let $|k| \rightarrow \infty$ changing $n$ and $\nu$ and preserving the relation $m=|k|(n \pm \nu)$. Then the limit of the right hand side of (7.14) exists and

$$
\lim _{|k|\left(n_{ \pm} \nu\right)=m,|k| \rightarrow \infty} \theta_{ \pm}(n, \nu ;|k|)=-\frac{1}{4 \pi} \int_{E_{3}} q(y) \exp \{ \pm i m \cdot y\} d y .
$$

Hence, we get the following uniqueness theorem for the scattering inverse problem, which slightly generalizes a result of Faddeev [4].

Theorem 7.2. If the potential $q(x)$ is assumed to satisfy condition $\left(\mathrm{A}_{1}\right)$, then it is uniquely determined by (7.15) from the scattering amplitude $\theta_{ \pm}(n, \nu ;|k|)$ given for large $|k|^{2}$ of the kinetic energy.

\section{References}

[1] Mochizuki, K., Eigenfunction expansions associated with the Schrödinger operator with a complex potential and the scattering inverse problem, Proc. Japan Acad. 43 (1967) 638-643.

[2] Povzner, A. Ya., On the expansion of arbitrary functions in characteristic functions of the operator $-\Delta u+c u$, Math. Sb. 32 (74) (1953), 109-156 (Russian).

[3] - On the expansion in terms of the eigenfunctions of the Schrödinger equation, Dokl. Akad. Nauk SSSR 104 (3) (1955), 360-363 (Russian).

[4] Faddeev, L. D., The uniqueness of solutions for the scattering inverse problem, Vestnik Leningrad Univ. 7 (1956), 126-130 (Russian).

[5] Ikebe, T., Eigenfunction expansions associated with the Schrödinger operators and their applications to the scattering theory, Arch. Rat. Mech. Anal. 5 (1960), $1-34$. 
[6] - On the phase-shift formula for the scattering operator, Pacific J. Math. 15 (1965), 511-523.

[7] Gohberg, I. C. and M. G. Krein, The basic propositions on defect numbers, root numbers and indices of linear operators, Uspehi Math. Nauk 12 (1957), 43-118 =Amer. Math. Soc. Transl. 13 (2) (1960), 185-264.

[8] Schechter, M., Fredholm operators and the essential spectrum, Preprint, Courant Institute, New York University, 1965.

[9] Eidus, D. M., On the principle of limiting absorption, Math. Sb. 57 (1962), 13-44 (Russian).

[10] Schwartz, J., Some non-selfadjoint operator, Comm. Pure Appl. Math. 13 (1960), 609-639.

[11] Pavlov, B. S., The non-selfadjoint Schrödinger operator, Topics Math. Phys. 1 (1967) $83-113=$ Special research report, translated from Russian; Consultants Bureau.

[12] Mochizuki, K., On the large perturbation by a class of non-selfadjoint operators, J. Math. Soc. Japan 19 (1967), 123-158.

[13] Kato, T., Wave operators and similarity for some non-selfadjoint operators, Math. Ann. 162 (1966), 258-279.

[14] — Purturbation theory for linear operators, Springer, 1966.

[15] - Growth properties of solutions of the reduced wave equation with a variable coefficient, Comm. Pure Appl. Math. 12 (1959), 403-425.

[16] Kuroda, S. T., An abstruct stationary approach to perturbation of continuous spectra and the scattering theory, J. Analyse Math. (to appear).

[17] - A stationary approach in the theory of scattering and eigenfunction expansions I, Sugaku 18 (1966), 74-85; II (1966) 137-144 (Japanese).

[18] Mizohata, S., Theory of partial differential equations, Iwanami, Tokyo, 1965 (Japanese).

[19] Ladyzenskaja, O. A., On the principle of limiting amplitude, Uspehi Math. Nauk 12 (1957), 161-164.

[20] Kiyama, S., On the exponential decay of solutions for the wave equation with the potential function, Osaka J. Math. 4 (1967), 15-35.

[21] Lax, P. D. and R. S. Phillips, The acoustic equation with an indefinite energy form and the Schrödinger equation, J. Functional Analysis 1 (1967), 37-83. 